\title{
SOME RESULTS ON THE SARKOVSKII PARTIAL ORDERING OF PERMUTATIONS
}

\author{
IRWIN JUNGREIS
}

\begin{abstract}
If $\pi$ is a cyclic permutation and $x$ is a periodic point of a continuous function $f: \mathbf{R} \mapsto \mathbf{R}$ with $\operatorname{period}(x)=\operatorname{order}(\pi)=n$, then we say that $x$ has type $\pi$ if the orbit of $x$ consists of points $x_{1}<x_{2}<\cdots<x_{n}$ with $f\left(x_{i}\right)=x_{\pi(i)}$. In analogy with Sarkovskii's Theorem, we define a partial ordering on cyclic permutations by $\theta \rightarrow \pi$ if every continuous function with a periodic point of type $\theta$ also has a point of type $\pi$. In this paper we examine this partial order form the point of view of critical points, itineraries, and kneading sequences. We show that $\theta \rightarrow \pi$ if and only if the maxima of $\theta$ are "higher" and the minima "lower" than those of $\pi$, where "higher" and "lower" are precisely defined in terms of itineraries. We use this to obtain several results about $\rightarrow$ : there are no minimal upper bounds; if $\pi$ and $\theta$ have the same number of critical points (or if they differ by 1 or sometimes 2 ), then $\theta \rightarrow \pi$ if and only if $\theta \rightarrow \pi_{*}$ for some period double $\pi_{*}$ of $\pi$; and finally, we prove a conjecture of Baldwin that maximal permutations of size $n$ have $n-2$ critical points, and obtain necessary and sufficient conditions for such a permutation to be maximal.
\end{abstract}

\section{SUMMARY OF NOTATION}

[n] The integers from 1 to $n$.

$C^{n} \quad$ The set of cyclic permutations of $[n]$.

type $\pi \quad$ A periodic point has type $\pi$ if $\pi$ describes the permuting of its orbit.

u.o.p.b. Unique order preserving bijection.

$\theta \rightarrow \pi$ Every function with a point of type $\theta$ has a point of type $\pi$.

$f_{\pi}$ The piecewise linear function connecting the dots $(i, \pi(i))$.

$z(\theta) \pm$ the number of critical points of $\theta,+$ if $\theta$ starts out increasing.

type $z \quad \theta$ has type $z$ if $z=z(\theta)$.

$z \quad z+\operatorname{sign}(z)=\operatorname{sign}(z)(1+|z|)$

$z^{-}-z^{+}$.

$I^{z} \quad$ Finite or infinite sequences of $L_{1}, \ldots, L_{|z|+1}, C_{1}, \ldots, C_{|z|}$.

$I_{\infty}^{z} \quad$ Infinite sequences of $L_{1}, \ldots, L_{|z|+1}$.

$A_{[m]} A_{0} A_{1} \cdots A_{m}$, where $A=A_{0} A_{1} \cdots$.

$S(A) \quad$ The shift of $A: A_{1} A_{2} \cdots$.

Received by the editors December 16, 1987 and, in revised form, April 30, 1989.

1980 Mathematics Subject Classification (1985 Revision). Primary 58F13, 54H20; Secondary 58F08, 70K50. 
$A^{\infty} \quad A A A \cdots$.

$|A|$ The number of terms in $A$.

odd $L_{i}$ is odd if $i$ is odd and $z<0$ or $i$ is even and $z>0$. A sequence is odd if it contains an odd number of odd terms.

$A$ has type $\theta \quad A$ is a periodic sequence which has type $\theta$ when considered as a periodic point of $S$.

$C_{i}(f)$ The $i$ th critical point of $f$.

$L_{i}(f)$ The interval $\left(C_{i-1}, C_{i}\right)$.

$i_{+} \quad$ The identity map from $I^{z}$ to $I^{z^{+}}$.

$i_{-} \quad$ The map from $I^{z}$ to $I^{z^{-}}$taking $C_{i}$ to $C_{i+1}$ and $L_{i}$ to $L_{i+1}$.

$I_{f}(x), I(x)$ The itinerary of $x$ under $f$.

$I_{f}^{+}(x), I^{+}(x) \quad \lim _{y \rightarrow x^{+}} I(y)$.

$I_{\theta}, L_{i}(\theta)$, etc. $I_{f_{\theta}}, L_{i}\left(f_{\theta}\right)$, etc.

$F(\theta) \quad I_{\theta}^{+}(1)$

$<_{i}<$ if $L_{i}$ is even and $>$ if $L_{i}$ is odd.

$A_{(i)} \quad$ The largest (w.r.t. $<_{i}$ ) shift of $A$ that follows $L_{i}$ or $L_{i+1}$.

$A$ is $i$-maximal $A=A_{(i)}$.

$\theta_{(i)} F(\theta)_{(i)}$.

$\ll \quad I \ll J$ if $I_{(i)} \leq_{i} J_{(i)}$ for all $i$.

period double $\theta$ is a period double of $\pi$ if $\theta(\{2 i-1,2 i\})=\{2 \pi(i)-$ $1,2 \pi(i)\}$ for all $i$.

$S(\theta) \quad$ The set of sequences in $I_{\infty}^{z}$ formed from $I_{\theta}(1)$ by changing $C_{i}$ to $L_{i}$ or $L_{i+1}$.

$S^{\prime}, S^{\prime \prime}, S^{\prime \prime \prime} \quad$ The sets of sequences like $S(\theta)$ but in $I^{z^{+}}, I^{z^{-}}$, and $I^{z^{+-}}$.

$P(z) \quad$ The set of cyclic permutations of type $z$.

$\left.\theta\right|_{U}$ The permutation of $U$ that takes each element of $U$ to the next one to appear in its $\theta$ orbit.

\section{INTRODUCTION}

Define $[n]=\{i \in \mathbf{Z}: 1 \leq i \leq n\}$ and $C^{n}=$ the set of cyclic permutations of $[n]$. We will usually assume that $n \geq 3$. Greek letters will always denote elements of $C^{n}$. If $\pi \in C^{n}, S$ an interval of $\mathbf{R}$, and $f: S \mapsto S$, then a periodic point $x$ of $f$ with smallest period $n$ is of type $\pi$ if the orbit of $x$ consists of points $x_{1}<x_{2}<\cdots<x_{n}$ with $f\left(x_{i}\right)=x_{\pi(i)}$. Otherwise put, if $g$ is the unique order preserving bijection (u.o.p.b.) from the orbit of $x$ to $[n]$, then $\pi=g \circ f \circ g^{-1}$.

If $\pi \in C^{n}$ and $\theta \in C^{m}$, we say $\theta \rightarrow \pi$ if every continuous function with a periodic point of type $\theta$ has a point of type $\pi$. Then $\rightarrow$ gives a reflexive partial order [1, Theorem 1.4]. The goal of this paper is to further understand this partial ordering.

Several authors have studied this partial ordering on permutations. See [1] for an overview, [2] for a discussion of immediate successors and period doubles, 
[8] for a description of minimal orbits, and $[3,4,5]$ for other results.

In $\S 2$ we give the notation for the rest of the paper. $\S 3$ deals with period doubles. In $\S 4$, we prove a theorem similar to $[7, \S 5$, second theorem] that gives conditions for a function with given kneading sequence to have a point with given itinerary. We use this theorem to produce an algorithm for testing if $\theta \rightarrow \pi$ using itineraries. $\S 5$ deals with the possible itineraries that a periodic point of given type can have.

In $\S 6$ we find a simple test for $\theta \rightarrow \pi$ when $\theta$ and $\pi$ have approximately the same number of critical points. We also prove under certain hypothesis that if $\theta \rightarrow \pi$ then $\theta \rightarrow \pi_{*}$ for some period double $\pi_{*}$ of $\pi . \S 7$ proves a theorem needed for the results of $\S 6$.

In $\S 8$ we prove that there are no minimal upper bounds for the partial ordering $\rightarrow$. Finally, in $\S 9$ we prove a conjecture of Baldwin that maximal permutations of size $n$ have $n-2$ critical points, and obtain necessary and sufficient conditions for such a permutation to be maximal.

\section{NOTATION AND PRELIMINARIES}

Define $f_{\theta}:[1, n] \mapsto[1, n]$, the primitive function of $\theta$, by $f_{\theta}(i)=\theta(i)$ for $i \in[n]$, and $f_{\theta}$ is the piecewise linear function "connecting the dots" $(i, \theta(i))$. In [1] it is shown that $\theta \rightarrow \pi$ if and only if $f_{\theta}$ has a periodic point of type $\pi$.

We define a critical point of a permutation $\pi \in C^{n}$ to be an integer $i$, with $2 \leq i \leq n-1$, such that $(\pi(i)-\pi(i-1))(\pi(i+1)-\pi(i))<0$, i.e., $i$ is an extreme point of $f_{\pi}$. Define $z=z(\theta)$ to be plus or minus the number of critical points of $\theta$, with $z(\theta)$ positive if $\theta(2)>\theta(1)$. We then say that $\theta$ has type $z$, and will usually denote $|z|=k$.

When dealing with permutations of type $z$, we will often consider permutations with exactly one more critical point. These have type $z+\operatorname{sign}(z)$ and $-z-\operatorname{sign}(z)$. To condense the notation, we define for $z \in \mathbf{Z} \backslash\{0\}$, $z^{+}=z+\operatorname{sign}(z)$ and $z^{-}=-z-\operatorname{sign}(z)$.

For $z \in \mathbf{Z} \backslash\{0\}, k=|z|$, define $I^{z}=\{$ finite or infinite sequences of symbols $\left.L_{1}, L_{2}, \ldots, L_{k+1}, C_{1}, C_{2}, \ldots, C_{k}\right\}$. If $A \in I^{z}$ define $A_{i}$ to be the $i+1$ st element of $A$, so $A_{0}$ is the first. Define $A_{[m]}=A_{0} A_{1} \cdots A_{m}$. Define the shift $S(A)$ to be the sequence $A_{1} A_{2} \cdots$. If $A, B \in I^{z}$ and $A$ is finite we will use $A B$ to denote the concatenation of $A$ and $B$, and $A^{\infty}$ to denote $A A A \cdots$. If $A$ is finite and $B=A C$ for some sequence $C$, we say that $A$ is a prefix of $B$. If $A$ is a prefix of $S^{j}(B)$ for some $j$ then $A$ is a substring of $B$. Define $I_{\infty}^{z}$ to be the subset of $I^{z}$ consisting of infinite sequences containing no $C_{i}$. For $A \in I^{z}$ finite define $|A|=$ the number of terms in $A$.

Define $L_{1}<C_{1}<L_{2}<\cdots<C_{k}<L_{k+1}$. Define $L_{i}$ to be odd if $i$ is odd and $z<0$ or if $i$ is even and $z>0$, and $L_{i}$ to be even otherwise. A sequence $L_{i_{1}} L_{i_{2}} \cdots L_{i_{m}}$ is odd if it contains an odd number of odd terms. Order the elements of $I^{z}$ as follows: Let $A \neq B \in I^{z}$. If $A$ or $B$ is a prefix of the other or if they contain a common prefix that contains some $C_{i}$, then $A$ and $B$ are 
incomparable. Otherwise there is an $m$ with $A_{[m-1]}=B_{[m-1]}, A_{m} \neq B_{m}$, and $A_{[m-1]}$ contains no $C_{i}$. Then $A$ and $B$ have the same ordering as $A_{m}$ and $B_{m}$ if and only if $A_{[m-1]}$ is even. Let $A \in I^{z}, \theta \in C^{n}$. We say that $A$ has type $\theta$ if $A$ has exact period $n$ and $\theta=g \circ S \circ g^{-1}$, where $g$ is the u.o.p.b. from $\left\{S^{i}(A)\right\}$ to $[n]$.

We define $i_{+}: I^{z} \mapsto I^{z^{+}}$and $i_{-}: I^{z} \mapsto I^{z^{-}}$to be the natural order preserving embeddings, i.e., $i_{+}$is the identity map, and $i_{-}$is defined as follows: for each $j, i_{-}\left(C_{j}\right)=C_{j+1}, i_{-}\left(L_{j}\right)=L_{j+1}$, and $i_{-}\left(A_{0} A_{1} \cdots\right)=i_{-}\left(A_{0}\right) i_{-}\left(A_{1}\right) \cdots$. Note that $i_{+}$and $i_{-}$are order preserving.

A continuous function $f:[a, b] \mapsto[a, b]$ is said to be piecewise monotone of type $z$ with $|z|=k$ if there are points $C_{1}, C_{2}, \ldots, C_{k}$ with $a=C_{0}<$ $C_{1}<\cdots<C_{k}<C_{k+1}=b$ such that $f$ is strictly monotone on $\left[C_{i}, C_{i+1}\right]$ for $0 \leq i \leq k$, not monotone on any larger intervals, and $\left.f\right|_{\left[C_{0}, C_{1}\right]}$ is increasing if and only if $z$ is positive. Call $C_{i}=C_{i}(f), i \in[k]$, the critical points of $f$ and define for $i \in[k+1], L_{i}=L_{i}(f)=$ the interval $\left(C_{i-1}, C_{i}\right)$ if $i \neq 1$ or $k+1, L_{1}=\left[a, C_{1}\right)$, and $L_{k+1}=\left(C_{k}, b\right]$. For $x \in[a, b]$ define the itinerary of $x, I_{f}(x)=I(x) \in I^{z}$, by

$$
I(x)_{i}= \begin{cases}C_{j} & \text { if } f^{i}(x)=C_{j}, j \in[k], \\ L_{j} & \text { if } f^{i}(x) \in L_{j} .\end{cases}
$$

The sequence $I(x)$ and the ordering of sequences have been defined so that $x \leq y$ implies $I(x) \leq I(y)$ and $I_{f}(f(x))=S\left(I_{f}(x)\right)$.

For $\theta \in C^{n}$ define $L_{i}(\theta), C_{i}(\theta)$, and $I_{\theta}(x)$ to be $L_{i}\left(f_{\theta}\right), C_{i}\left(f_{\theta}\right)$, and $I_{f_{\theta}}(x)$, respectively.

Note that if $\theta$ has type $z$ and $f$ is a piecewise monotone function with a point of type $\theta$ then clearly $z(f)=z$ or $|z(f)|>|z|$. In particular, if $\pi \rightarrow \theta$ then $z(\pi)=z(\theta)$ or $|z(\pi)|>|z(\theta)|$.

Define $I^{+}(x)=\lim _{y \rightarrow x^{+}} I(y)$ (in the topology of termwise convergence). Clearly $I^{+}(x)$ is defined and is in $I_{\infty}^{z}$. Define $I^{-}(x)$ likewise. We denote $F(\theta)=I_{\theta}^{+}(1)$, the fundamental sequence of $\theta$. Note that if $x$ is a critical point, then $S\left(I^{+}(x)\right)=S\left(I^{-}(x)\right)$.

To avoid repeated statements of the form " $A<B$ if $L_{i}$ is even and $B<A$ if $L_{i}$ is odd" we introduce the following notation: when we are dealing with functions of type $z$ or sequences in $I^{z}$ we will use $<_{i}$ to mean $<$ if $L_{i}$ is even and to mean $>$ otherwise. Note that $<_{i}$ and $<_{i+2}$ mean the same thing. Define $>_{i}, \leq_{i}$, and $\geq_{i}$ in the obvious way. This should be thought of as a notational convenience, rather than a new order relation. We will use it anywhere the symbol < could be used, not just for comparisons in a particular set. As some examples of the use of $<_{i}$ note that: if $A<B$ then $L_{i} A<_{i} L_{i} B$; for $0 \leq i \leq k+1, C_{i}(f)$ is a local maximum with respect to $<_{i} ; f$ is increasing on $L_{i}$ and decreasing on $L_{i+1}$ with respect to $<_{i}$.

Given a sequence $A \in I^{z}$, for $0 \leq i \leq k+1$ define the ith critical value of $A$, 
denoted $A_{(i)}$, to be the supremum with respect to $<_{i}$ of those shifts $S^{j+1}(A)$ for which $A_{j}=L_{i}$ or $L_{i+1}$, and $A_{(i)}$ is undefined if $A$ contains no $L_{i}$ 's or $L_{i+1}$ 's. Intuitively, if $A=I_{f}(x)$ then $A_{(i)}$ is the itinerary of the image under $f$ of the point of the orbit of $x$ that is closest to $C_{i}$. In other words $A_{(i)}$ is the height of the $i$ th critical point. For $\theta \in C^{n}$ define $\theta_{(i)}=I_{\theta}^{+}(1)_{(i)}=F(\theta)_{(i)}$. Clearly $\theta_{(i)}=S\left(I_{\theta}^{+}\left(C_{i}\right)\right)=S\left(I_{\theta}^{-}\left(C_{i}\right)\right)$. For $0 \leq i \leq k+1$ define a periodic sequence $A \in I^{z}$ to be $i$-maximal if $A=A_{(i)}$. Note that $\theta_{(i)}$ is always $i$-maximal.

Define a partial ordering $\ll$ on $I_{\infty}^{z},|z|=k$, as follows: if $I, J \in I_{\infty}^{z}$ define $I \ll J$ to mean that for $1 \leq i \leq k$, if $I_{(i)}$ is defined then so is $J_{(i)}$ and $I_{(i)} \leq_{i} J_{(i)}$. Intuitively, $I \ll J$ means that all of the maxima of $I$ are lower and all the minima higher than the corresponding extrema of $J$.

\section{Period doubles}

A period double is a permutation $\theta \in C^{n}$ that is divisible by 2, in Baldwin's notation, i.e., $n$ is even and there is a $\theta_{*} \in C_{n / 2}$ with $\theta(\{2 i-1,2 i\})=$ $\left\{2 \theta_{*}(i)-1,2 \theta_{*}(i)\right\}$ for $i \in[n / 2]$. We say $\theta$ is a period double of $\theta_{*}$.

Lemma 3.1. If $f_{\theta}$ has two distinct points $x$ and $y$ with $I_{\theta}(x)=I_{\theta}(y)$ then $\theta$ is a period double. If $x$ is periodic then the integer part of $x=$ the integer part of $y=m$, an odd integer, $I_{\theta}^{+}(m)=I_{\theta}(z)=I_{\theta}^{-}(m+1)$ for all $z \in(m, m+1)$, and each such $z$ is part of a periodic orbit of type $\theta$, except $m+\frac{1}{2}$ which has type $\theta / 2$.

Proof. Let $T=\left\{z: I_{\theta}(z)=I_{\theta}(x)\right\} . T$ is connected since $I_{\theta}$ is monotone, and $T$ has positive length since it includes $x$ and $y$. Since all points of $T$ have the same itinerary, $f^{j}(T)$ contains no critical points. Hence $\left.f^{j}\right|_{T}$ is monotone. Since $\left|f_{\theta}^{\prime}\right| \geq 1, \mu\left(f^{j+1}(T)\right) \geq \mu\left(f^{j}(T)\right)$. (Here $\mu$ denotes Lebesgue measure.) Hence for some $j$ and $i, f^{j}(T)$ and $f^{i}(T)$ are not disjoint. Assume without loss of generality that $i=0$. Otherwise take new $x$ and $y$ in $f^{i}(T)$. Then $I_{\theta}(x)=S^{j}\left(I_{\theta}(x)\right)$. Since every point of $f^{j}(T)$ has itinerary $S^{j}\left(I_{\theta}(x)\right)$, $f^{j}(T) \subset T$. Since $\mu\left(f^{j}(T)\right) \geq \mu(T), f^{j}(T)=T$. Hence $\left|f_{\theta}^{\prime}\right|=1$ on all the unit intervals with integer endpoints containing a point of the orbit of $f^{i}(x)$. Hence all points of these intervals have the same itinerary, and $f^{j}$ is an isometry on these intervals. Hence all the images of some integer and its successor remain unit distance apart. This implies that $\theta$ is a period double. The rest is obvious.

Theorem 3.2. If $\theta \in C^{n}$ then $F(\theta)$ has exact period $n$ unless $\theta$ is a period double, in which case it has exact period $n / 2$.

Proof. Since $\theta^{n}(1)=1$ and 1 is the global minimum of $f_{\theta}$, if $x$ is a little bigger than 1 then $\theta^{n}(x)$ will also be a little bigger than 1 . Hence $S^{n}(F(\theta))=$ $F(\theta)$. Let $m$ be the period of $F(\theta)$, so $S^{m}(F(\theta))=F(\theta)$. But $S^{m}(F(\theta))=$ $I_{\theta}^{ \pm}\left(\theta^{m}(1)\right)$. If $m<n$ then all points between 1 and $\theta^{m}(1)$ have the same 
itinerary. By Lemma 3.1, $\theta$ is a period double. In this case $f^{m}$ is monotone on $\left[1, \theta^{m}(1)\right]$ so it can have no periodic point of order more than 2 . Since 1 has period $n / m$ under $f^{m}, m \geq n / 2$. On the other hand, if $\theta$ is a period double then $\theta^{i}(1)$ and $\theta^{i}(2)$ are always consecutive. So $\theta^{n / 2}[1,2]=[1,2]$ and $\theta^{n / 2}(1)=2$, so $S^{n / 2}(F(\theta))=I_{\theta}^{-}(2)=F(\theta)$, so $F(\theta)$ has period $n / 2$.

Define a periodic sequence $A^{\infty} \in I_{\infty}^{z}$ with $A$ of minimal length to be odd if $A$ is odd and even otherwise.

Theorem 3.3. $F(\theta)$ is odd if and only if $\theta$ is a period double. If $\theta$ is a period double, say of $\theta_{*}$, then $F(\theta)$ has type $\theta_{*}$; otherwise it has type $\theta$.

Proof. Since $f_{\theta}^{n}(1)=1,1$ is a local minimum for $f_{\theta}^{n}$. Hence $F(\theta)_{[n-1]}$ is even. If $\theta$ is not a period double, then the smallest repeating block of $F(\theta)$ is $F(\theta)_{[n-1]}$, which is even. If $\theta$ is a period double, its smallest repeating block is $F(\theta)_{[n / 2-1]}$. Since $\theta^{n / 2}(1)=2$ and $f_{\theta}^{n / 2}$ maps [1, 2] to itself, $f_{\theta}^{n / 2}(x)$ is decreasing for $x$ slightly larger than 1 . Hence $F(\theta)_{[n / 2-1]}$ is odd. If $\theta$ is not a period double, then all the shifts $S^{i}(F(\theta)), 0 \leq i<n$, are distinct. Since $S^{i}(F(\theta))$ is $I_{\theta}^{+}\left(\theta^{i}(1)\right)$ or $I_{\theta}^{-}\left(\theta^{i}(1)\right)$, and $I_{\theta}^{ \pm}\left(\theta^{i}(1)\right)<I_{\theta}^{ \pm}\left(\theta^{j}(1)\right)$ if and only if $\theta^{i}(1)<\theta^{j}(1)$, we have $F(\theta)$ has type $\theta$. If $\theta$ is a period double, then $F(\theta)=I_{\theta}(3 / 2)$, a point of type $\theta_{*}$, and $F(\theta)$ has exact period $n / 2=\operatorname{ord}\left(\theta_{*}\right)$, so $I_{\theta}(3 / 2)$ has type $\theta_{*}$.

Remark. We say that $\pi$ is an immediate successor if $\theta$ if $\pi \rightarrow \theta$ and $\pi \rightarrow$ $\phi \Rightarrow \pi=\phi$ or $\theta \rightarrow \phi$. It is shown in [2, Theorem 1.12] that $\pi$ is an immediate successor of $\theta$ if and only if $\pi$ is a period double of $\theta$.

We will have more to say about period doubles in $\S 6$.

\section{EXISTENCE OF POINTS WITH GIVEN ITINERARY}

Theorem 4.1. Let $A \in I_{\infty}^{z}, k=|z|$, and $f$ be piecewise monotone of type $z$. If for all $j \geq 0$

$$
A_{j}=L_{i} \Rightarrow I^{+}\left(C_{i-1}\right)<S^{j}(A)<I^{-}\left(C_{i}\right)
$$

then there is an $x$ with $I(x)=A$. Conversely, if $I(x) \in I_{\infty}^{z}$ and $I(x)_{j}=L_{i}$ then $I^{+}\left(C_{i-1}\right) \leq S^{j}(A) \leq I^{-}\left(C_{i}\right)$.

Remark. This is a slight generalization of [7, $\S 5$, second theorem].

Proof. The converse part is obvious from the fact that $x \leq y$ implies that $I(x) \leq I(y)$. Suppose that $A$ has the given properties. Let $S=\{x: I(x) \leq A\}$ and $T=\{x: I(x) \geq A\}$. By assumption, $a \in S$ and $b \in T$ (since $I(a) \leq$ $I^{+}\left(C_{i-1}\right) \leq I^{-}\left(C_{i}\right) \leq I(b)$ for all $\left.i\right)$, so $S$ and $T$ are nonempty. We will show that they are open (as subsets of $[a, b]$ ). Since $S$ is clearly connected, it suffices to show that $y \in S$ implies that $y+\varepsilon \in S$ for small $\varepsilon>0$. Let $y \in S$ and choose $j$ with $I(y)_{[j-1]}=A_{[j-1]}$ but $I(y)_{j} \neq A_{j}=L_{i}$. Assume $A_{[j-1]}$ is even. Then $I(y)_{j}<A_{j}=L_{i}$. Since $\left\{z: I(z)_{[j-1]}=A_{[j-1]}, I(z)_{j}<C_{i-1}\right\}$ 
is open, we need only consider the case $I(y)_{j}=C_{i-1}$. Since $A_{[j-1]}$ is even, for $\varepsilon$ sufficiently small $f^{j}(y+\varepsilon)$ will be slightly larger than $f^{j}(y)=C_{i-1}$, so $I(y+\varepsilon)$ will agree with $A_{[j-1]} I^{+}\left(C_{i-1}\right)$ to any desired number of terms. Since by assumption $S^{j}(A)>I^{+}\left(C_{i-1}\right)$ so $A=A_{[j-1]} S^{j}(A)>A_{[j-1]} I^{+}\left(C_{i-1}\right)$, we have $A>I(y+\varepsilon)$ for $\varepsilon$ sufficiently small. If $A_{[j-1]}$ is odd, then $I(y)_{j}>A_{j}=L_{i}$ and we only need consider $I(y)_{j}=C_{i}$. For $\varepsilon$ small, $I(y+\varepsilon)$ will agree with $A_{[j-1]} I^{-}\left(C_{i}\right)$ for any desired number of terms. Then $S^{j}(A)<I^{-}\left(C_{i}\right)$ gives $A>I(y+\varepsilon)$. The proof for $T$ is similar. Since $[a, b]$ is not the union of two disjoint nonempty open sets, it contains $x$ with $x \in S \cap T$, i.e., $I(x)=A$.

Theorem 4.2. Let $f$ be piecewise monotone. If $x$ is a periodic point of $f$ of type $\pi$ and $I_{f}(x)$ has exact period $n=\operatorname{ord}(\pi)$ then $I(x)$ has type $\pi$. In particular, if $f=f_{\theta}$ and $\pi \neq \theta$ then $I_{\theta}(x)$ has type $\pi$. Conversely, if $f$ has a point $x$ with $I(x)$ of type $\pi$ then $f$ has a periodic point of type $\pi$.

Proof. The first part follows from the fact that $I(x)<I(y)$ implies $x \leq y$, since $I(x)$ has exact period $n$. If $I_{\theta}(x)$ has period smaller than $n$ then there is a $y$ in the orbit of $x$ with $I(y)=I(x)$. By Lemma 3.1 $x$ (or $y$ ) has type $\theta$, contradicting the fact that $\theta \neq \pi$.

For the converse we note that the proof of Lemmas II.3.1 and II.3.2 of [6], which deal with unimodal functions, goes through exactly to the case of piecewise monotone functions. This shows that as $j \rightarrow \infty, f^{j n}$ converges to a periodic point $y$ of period $n$ or $2 n$. In the latter case $f^{n}(y)$ and $y$ have the same itinerary, as do all points between them. Since $f^{n}$ exchanges $y$ and $f^{n}(y)$, it must have a fixed point between them. Thus in either case we get a point of period $n$. This point clearly has type $\pi$.

Theorem 4.3. Let $\theta \in C^{n}$ have type $z, k=|z|$, and let $A \in I_{\infty}^{z}$ be periodic, with $A \notin\left\{L_{1}^{\infty}, L_{k+1}^{\infty},\left(L_{1} L_{k+1}\right)^{\infty},\left(L_{k+1} L_{1}\right)^{\infty}\right\}$. Then $f_{\theta}$ has a point with itinerary $A$ if and only if either $F(\theta) \gg A$ with $A$ not a shift of $F(\theta)$, or $\theta$ is a period double and $A$ is a shift of $F(\theta)$.

Proof. First, suppose that $f_{\theta}$ has a point $x$ with itinerary $A$ and that $A_{(i)}$ is defined. Then for some $j, S^{j}(A)=L_{i} A_{(i)}$ or $L_{i+1} A_{(i)}$. Then by Theorem 4.1, $S^{j}(A) \leq I^{-}\left(C_{i}\right)$ in the former case and $I^{+}\left(C_{i}\right) \leq S^{j}(A)$ in the latter. Either case then gives

$$
A_{(i)}=S^{j+1}(A) \leq_{i} S\left(I^{+}\left(C_{i}\right)\right)=S\left(I^{-}\left(C_{i}\right)\right)=\theta_{(i)} .
$$

If $A_{(i)}=\theta_{(i)}$ then $A$ is a shift of $F(\theta)$. If there is a point $x$ with itinerary $F(\theta)$ then $x>1$, since $I(1)$ includes $C$ 's. So all points between 1 and $x$ have the same itinerary. By Lemma 3.1, $\theta$ must be a period double.

Conversely, suppose that $\theta_{(i)}>_{i} A_{(i)}$ for al $i$ for which $A_{(i)}$ is defined. We need only verify that equation (1) of Theorem 4.1 is satisfied. Suppose that $A_{j}=L_{i}$ and assume $i \neq 1$ or $k+1$. Then by definition of $i$-maximal, $A_{(i)} \geq_{i} S^{j+1}(A)$, so $\theta_{(i)}>_{i} S^{j+1}(A)$. If $L_{i}$ is even this gives $\theta_{(i)}>S^{j+1}(A)$ so 
$L_{i} \theta_{(i)}>L_{i} S^{j+1}(A)=S^{j}(A)$, so $I^{-}\left(C_{i}\right)>S^{j}(A)$. If $L_{i}$ is odd, $\theta_{(i)}<S^{j+1}(A)$, $L_{i} \theta_{(i)}>L_{i} S^{j+1}(A), I^{-}\left(C_{i}\right)>S^{j}(A)$. The proof that $I^{+}\left(C_{i-1}\right)<S^{j}(A)$ is similar: $\theta_{(i-1)}>_{i-1} A_{(i-1)} \geq_{i-1} S^{j+1}(A), L_{i} \theta_{(i-1)}<L_{i} S^{j+1}(A), I^{+}\left(C_{i-1}\right)<$ $S^{j}(A)$.

Now for $i=1$ or $k+1$, the above argument shows that $S^{j}(A)<I^{-}\left(C_{1}\right)$ if $i=1$ and $I^{+}\left(C_{k}\right)<S^{j}(A)$ if $i=k+1$. We need only show that $A_{j}=L_{1}$ implies $I^{+}(1)<S^{j+1}(A)$ and $A_{j}=L_{k+1}$ implies $S^{j+1}(A)<I^{-}(n)$. Since 1 is the global minimum of $f_{\theta}$ and $n$ is the global maximum, we have for all $i$

$$
I^{+}(1) \leq S\left(I^{+}\left(C_{i}\right)\right)=S\left(I^{-}\left(C_{i}\right)\right) \leq I^{-}(n) .
$$

We prove that if (1) holds for some $j$ then it holds for $j+1$. This is already known unless $A_{j+1}=L_{1}$ or $L_{k+1}$. Suppose $A_{j+1}=L_{1}$. We know that $S^{j+1}(A)<I^{-}\left(C_{1}\right)$. Rewriting (1) with $A_{j}=L_{i}, L_{i} S\left(I^{+}\left(C_{i-1}\right)\right)<L_{i} S^{j+1}(A)<$ $L_{i} S\left(I^{-}\left(C_{i}\right)\right)$, we see that

$$
S^{J+1}(A)>\min \left(S\left(I^{+}\left(C_{i-1}\right)\right), S\left(I^{-}\left(C_{i}\right)\right)\right) \geq I^{+}(1)=I^{+}\left(C_{0}\right),
$$

establishing (1) for $j+1$. The case $A_{j+1}=L_{k+1}$ is similar. This establishes (1) inductively for all $m \geq j$. By periodicity of $A$ this establishes it for all $m$. Thus we need only establish (1) for one value of $j$.

If $A_{j} \neq L_{1}$ or $L_{k+1}$ for some $j$, we are done, so assume that $A_{j}=L_{1}$ or $L_{k+1}$ for all $j$. First, we consider the case that $L_{1}$ is even. By assumption, $A \neq$ $L_{1}^{\infty}$ or $L_{k+1}^{\infty}$, so for some $j, A_{j}=L_{1}$ and $A_{j+1}=L_{k+1}$. But $A_{j}=L_{1}$ implies that $L_{1} S^{j+1}(A)=S^{j}(A)<I^{-}\left(C_{1}\right)$, which implies $S^{j+1}(A)<S\left(I^{-}\left(C_{1}\right)\right) \leq$ $I^{-}(n)$, so we are done. Similarly, if $L_{k+1}$ is even then $A_{j}=L_{k+1}, A_{j+1}=L_{1}$ imply $I^{+}(1) \leq S\left(I^{+}\left(C_{k}\right)\right)<S^{j+1}(A)$, so $j+1$ satisfies (1). In the remaining case, $L_{1}$ and $L_{k+1}$ are each odd. Our assumptions on $A$ imply that there is a $j$ with $A_{j}=A_{j+1}=L_{1}$ or $A_{j}=A_{j+1}=L_{k+1}$. In the former case $L_{1} S^{j+1}(A)=S^{j}(A)<I^{-}\left(C_{1}\right)$ which implies $S^{j+1}(A)>S\left(I^{-}\left(C_{1}\right)\right) \geq I^{+}(1)$. In the latter case $L_{k+1} S^{j+1}(A)>I^{+}\left(C_{k}\right)$ implies $S^{j+1}(A)<I^{-}(n)$.

Finally, if $\theta$ is a period double, then all points in $(1,2)$ have itinerary $F(\theta)$.

Corollary 4.4. Let $\theta \in C^{n}, n \geq 3$, be a permutation of type $z$, and let $\pi \neq \theta$. Then $f_{\theta}$ has a periodic point of type $\pi$ if and only if there is a periodic sequence $A \in I_{\infty}^{z}$ of type $\pi$ with $A \ll F(\theta)$.

Proof. Since $\theta$ is assumed to have period at least 3 , the cases $\pi=(1)$ and (12) are trivial. The four excluded possibilities for $A$ in the theorem cannot have type $\pi \neq(1)$ or (12). If there is an $A$ of type $\pi$ with $\theta \gg A$ then by the theorem, $f_{\theta}$ has a point with itinerary $A$. By Theorem $4.2, f_{\theta}$ has a periodic point of type $\pi$. If $\theta_{(i)}=A_{(i)}$ and $\theta$ is a period double, then again Theorems 
4.2 and 4.3 give the desired point. If $\theta$ is not a period double, then $\theta_{(i)}$ has type $\theta$, so $\theta_{(i)}=A_{(i)}$ implies $\theta=\pi$, contradicting our assumption.

Conversely, if $f_{\theta}$ has a point $x$ of type $\pi \neq \theta$, then by Theorem 4.2, $A=I(x)$ has type $\pi$, and by Theorem $4.3, \theta_{(i)}>_{i} A_{(i)}$ or $A$ is a shift of $F(\theta)$, hence $\theta_{(i)}=A_{(i)}$.

Corollary 4.4 can be used as an algorithm for deciding if $\theta \rightarrow \pi$, namely find all sequences $A$ of type $\pi$ in $I_{\infty}^{z(\theta)}$, and for each one check if $F(\theta) \gg A$. To estimate the computational complexity of this algorithm we must bound the number of such sequences.

Let $A$ have type $\pi, \operatorname{ord}(\pi)=n, \operatorname{ord}(\theta)=m, k=|z(\theta)|$, and let $g$ be the u.o.p.b. from the shifts of $A$ to [n]. For $i=1, \ldots, k+1$ let $a_{i}=$ $\max \left\{j: g^{-1}(j)_{0} \leq L_{i}\right\}$ and $a_{i}=0$ if this set is empty. Then $0 \leq a_{1} \leq a_{2} \leq \cdots \leq$ $a_{k+1} \leq n$ and $\left(a_{1}, a_{2}, \ldots, a_{k+1}\right)$ certainly determines $A$. Thus the number of possible $A$ 's is $O\left((n+1)^{k+1}\right)$. (Note that this also gives us an algorithm for listing them.) Checking if $\theta_{(i)} \geq_{i} A_{(i)}$ takes time $O(n+m)$, and we must do this for $k$ values of $i$. Thus we get an overall complexity of $O\left(k(n+m)(n+1)^{k+1}\right)$. Notice that for fixed $k$ this is a polynomial time algorithm. The usual Markov graph algorithm given in [1, Theorem 2.4] is exponential even for fixed $\theta$.

In $\S 6$ we show that if $z(\theta)=z(\pi), z(\pi)^{+}, z(\pi)^{-}$, or $z(\pi)^{+-}$then only one sequence $A$ needs to be checked. Thus with that restriction the algorithm becomes quadratic (even with no restriction on $k$ ).

\section{ITINERARIES OF A GIVEN TYPE}

We have seen that to determine if $\pi \rightarrow \theta$ we need to know the sequences of type $\theta$. Given $\theta \in C^{n}$ of type $z$ with $k|z|$, in this section we find all sequences of type $\theta$ in $I_{\infty}^{z}, I_{\infty}^{z^{+}}, I_{\infty}^{z^{-}}$, and $I_{\infty}^{z^{+-}}$.

Let $S=S(\theta)$ be the set of $2^{k}$ sequences in $I_{\infty}^{z}$ formed by the following procedure: starting with $I_{\theta}(1)$, for each $i=1, \ldots, k$ change all occurrences of $C_{i}$ to $L_{i}$ or $L_{i+1}$. Note that $F(\theta) \in S$.

Let $I_{\theta}^{\prime}(1)$ be the sequence formed as $I_{\theta}(1)$ was except that we consider $n$ to be $C_{k+1}$ rather than an element of $L_{k+1}$. Similarly, let $I_{0}^{\prime \prime}(1)$ be the sequence formed by considering 1 to be $C_{0}$, and form $I_{\theta}^{\prime \prime \prime}(1)$ by considering 1 to be $C_{0}$ and $n$ to be $C_{k+1}$. Thus $I_{\theta}^{\prime}(1), I_{\theta}^{\prime \prime}(1)$, or $I_{\theta}(1)$ can be obtained from $I_{\theta}^{\prime \prime \prime}(1)$ by changing al $C_{0}$ 's to $L_{1}$ 's, all $C_{k+1}$ 's to $L_{k+1}$ 's, or both, respectively.

Let $S^{\prime}, S^{\prime \prime}$, and $S^{\prime \prime \prime}$ be the sets of $2^{k+1}, 2^{k+1}$, and $2^{k+2}$ sequences in $I_{\infty}^{z^{+}}, I_{\infty}^{z^{-}}$, and $I_{\infty}^{z^{+-}}$, respectively, formed from $i_{+}\left(I_{\theta}^{\prime}(1)\right), i_{-}\left(I_{\theta}^{\prime \prime}(1)\right)$, and $i_{+} i_{-}\left(I_{\theta}^{\prime \prime \prime}(1)\right)$ by changing all occurrences of $C_{i}$ to $L_{i}$ or $L_{i+1}$ for $1 \leq i \leq k+1$, $1 \leq i \leq k+1$, and $1 \leq i \leq k+2$, respectively.

Lemma 5.1. Let $\theta \in C^{n}$ have type $z, k=|z|$. Then $F(\theta)$ is the minimum element of $S$ with respect to the partial order $\ll$, and likewise $i_{+}(F(\theta)), i_{-}(F(\theta))$, and $i_{+} i_{-}(F(\theta))$ are the minima of $S^{\prime}, S^{\prime \prime}$, and $S^{\prime \prime \prime}$ respectively. 
Proof. Clearly if $i_{+}(I) \ll i_{+}(J)$ or $i_{-}(I) \ll i_{-}(J)$ then $I \ll J$. Since $I \in S$ implies $i_{+}(I) \in S^{\prime}$, etc., it suffices to prove that $i_{+} i_{-}(F(\theta))$ is the minimum element of $S^{\prime \prime \prime}$.

Let $I=i_{+} i_{-}(F(\theta))$ and $I \neq J \in S^{\prime \prime \prime}$. Pick $i \in[k+2]$. Find the $r$ satisfying $I_{(i)[r-1]}=J_{(i)[r-1]}$ and $I_{(i) r} \neq J_{(i) r}$. Let $A=I_{(i)[r-1]}$ and $L_{s^{\prime}}=I_{(i) r}$. Recall that if $i_{+} i_{-}\left(I_{\theta}^{\prime \prime \prime}(1)\right)_{(i) r}=L_{j}$ then $I_{(i) r}=J_{(i) r}=L_{j}$ and if $i_{+} i_{-}\left(I_{\theta}^{\prime \prime \prime}(1)\right)_{(i) r}=C_{s}$ then $I_{(i) r}$ and $J_{(i) r}$ must each be $L_{s}$ or $L_{s+1}$. Since $I_{(i) r} \neq J_{(i) r}$ the latter case must occur.

We have that $I_{(i)}=i_{+} i_{-}\left(F(\theta)_{(i-1)}\right)$, and $i_{+} i_{-}\left(I_{\theta}^{\prime \prime \prime}(1)\right)_{(i) r}=C_{s}$ implies $I_{\theta}^{\prime \prime \prime}(1)_{(i-1) r}=C_{s-1}$, or equivalently, $\theta^{r}\left(C_{i-1}(\theta)\right)=C_{s-1}(\theta)$.

Suppose $C_{i-1}(\theta)$ is a local maximum for $f_{\theta}$. Then $f_{\theta}\left(C_{i-1} \pm \varepsilon\right)<f_{\theta}\left(C_{i-1}\right)$. Now we need to use the fact that $F(\theta)$ is (to any fixed number of terms) the itinerary of points sufficiently close to 1 . The $\theta$ orbit of a point close to 1 will include a point close to $C_{i-1}$. The following point will be less than $f_{\theta}\left(C_{i-1}\right)$. The sequence of intervals through which the orbit then passes is given by $i_{-}^{-1} i_{+}^{-1}\left(A L_{s^{\prime}}\right)$ after which the orbit is near $f_{\theta}\left(C_{s-1}\right)$. If $C_{s-1}(\theta)$ is a local maximum, the point of our orbit will be slightly less, in which case $f_{\theta}^{r+1}(x)$ must be increasing for $x$ slightly less than $f_{\theta}\left(C_{i-1}\right)$. Hence $I_{-}^{-1} i_{+}^{-1}\left(A L_{s^{\prime}}\right)$ is even (as a sequence in $I_{\infty}^{z}$ ) so $A L_{s^{\prime}}$ is even (as a sequence in $I_{\infty}^{z^{+-}}$).

Assuming as above that $C_{i-1}(\theta)$ and $C_{s-1}(\theta)$ are local maxima of $f_{\theta}$, we have $L_{s-1}$ is even in $I_{\infty}^{z}$, so $L_{s}$ is even in $I_{\infty}^{z^{+-}}$. Suppose $A$ is even. Then $L_{s^{\prime}}$ is even so $L_{s^{\prime}}=L_{s}$. Hence $I_{(i)}=A L_{s^{\prime}} \cdots=A L_{s} \cdots<A L_{s+1} \cdots=J_{(i)}$, so $I_{(i)}<_{i} J_{(i)}$. The cases $A$ odd and $C_{i-1}(\theta)$ or $C_{s-1}(\theta)$ minima are similar.

Lemma 5.2. If $\theta \in C^{n}$ has type $z, k=|z|$, and is not a period double, then all elements of $S, S^{\prime}, S^{\prime \prime}$, and $S^{\prime \prime \prime}$ have exact period $n$. If $\theta$ is a period double then $F(\theta), i_{+}(F(\theta)), i_{-}(F(\theta))$, and $i_{+} i_{-}(F(\theta))$ have exact period $n / 2$ and all others have exact period $n$.

Proof. If $I \in\left(S^{\prime} \backslash i_{+}(S)\right)$ then $I$ contains exactly one $L_{k+2}$ every $n$ terms, so it has exact period $n$. If $I \in i_{+}(S)$ then $I$ has the same period as $i_{+}^{-1}(I) \in S$. Similarly, the only elements of $S^{\prime \prime}$ and $S^{\prime \prime \prime}$ that might not have period $n$ are in $i_{-}(S)$ and $i_{+} i_{-}(S)$. So we need only show the result for $S$.

The statement concerning $F(\theta)$ is just Theorem 3.2, so we need only show that if $J \in S, J \neq F(\theta)$, then $J$ has exact period $n$.

Given $0<\varepsilon<1$ we construct a piecewise monotone function $g_{\varepsilon}$ of type $z$ with the following properties:

(i) $\lim _{\varepsilon \rightarrow 0} g_{\varepsilon}=f_{\theta}$.

(ii) $g_{\varepsilon}^{m}(1)=\theta^{m}$ (1) for all $m \in \mathbf{Z}$.

(iii) $I_{g_{e}}(1)=J$.

Define $g_{\varepsilon}=f_{\theta}$ on integers. This assures condition (ii). Suppose $C_{i}$ is a maximum. If the corresponding term in $J$ is $L_{i}$, then require $g_{\varepsilon}\left(C_{i}+\varepsilon\right)=$ $f_{\theta}\left(C_{i}\right)+\varepsilon$; otherwise require $g_{\varepsilon}\left(C_{i}-\varepsilon\right)=f_{\theta}\left(C_{i}\right)+\varepsilon$. If $C_{i}$ is a minimum, 
change those to $f_{\theta}\left(C_{i}\right)-\varepsilon$. Now make $g_{\varepsilon}$ the simplest piecewise linear function satisfying those requirements. Since $L_{i}\left(g_{\varepsilon}\right)=\left(C_{i-1} \pm \varepsilon, C_{i} \pm \varepsilon\right)$, and we have chosen the signs properly, condition (iii) holds. Condition (i) is obvious.

Suppose $S^{l}(J)=J$ for some $l<n$. Let $r=\theta^{l}(1)$. Then $I_{g_{e}}(r)=I_{g_{e}}(1)$. By monotonicity of $I_{g_{e}}, J=I_{g_{e}}(1)=I_{g_{e}}(x)$ for all $x \in[1, r]$. Let $U=$ $\left\{x: I_{g_{\varepsilon}}(x)=J\right\}$. Then $g_{\varepsilon}^{l}$ is monotone on $U$ and $g_{\varepsilon}^{l}(U) \subset U$. But monotone functions on $\mathbf{R}$ cannot have periodic points of order greater than 2. Thus $g_{\varepsilon}^{l}(1)=1$ or $g_{\varepsilon}^{2 l}(1)=1$. The former case leads to the contradiction $l=n$. Otherwise $l=n / 2, r=\theta^{n / 2}(1), 1=\theta^{n / 2}(r)$, so $g_{\varepsilon}^{n / 2}$ maps $[1, r]$ to $[1, r]$ and is monotone. Taking the limit as $\varepsilon \rightarrow 0$ we see that $f_{\theta}^{n / 2}$ maps $[1, r]$ to $[1, r]$ and is monotone. Hence all points in $(1, r)$ have the same $f_{\theta}$ itinerary. By Lemma 3.1, $\theta$ is a period double, in which case $F(\theta)=I$ also has period $n / 2$.

Suppose $J$ differs from $I$ in the $s$ th term. Then $F(\theta)_{s}=C_{m}$ for some $m$. Then one of $I_{s}$ and $J_{s}$ is $L_{m}$ and the other is $L_{m+1}$. But since each has period $n / 2$, the same is true of $I_{s+n / 2}$ and $J_{s+n / 2}$. But then $F(\theta)_{s+n / 2}=C_{m}$, contradicting the fact that $C_{m}$ has exact period $n$.

Theorem 5.3. Let $\theta \in C^{n}$ have type $z, k=|z|$. Then the set of sequences of $I_{\infty}^{z}, I_{\infty}^{z^{+}}, I_{\infty}^{z^{-}}$, and $I_{\infty}^{z^{+-}}$of type $\theta$ are $S, S^{\prime}, S^{\prime \prime}$, and $S^{\prime \prime \prime}$ respectively, unless $\theta$ is a period double, in which case the result is the same except that $F(\theta)$, $i_{+}(F(\theta)), i_{-}(F(\theta))$, and $i_{+} i_{-}(F(\theta))$ do not have type $\theta$. (Here we consider a periodic sequence and its shifts to be the same.)

Proof. First we show that all the given sequences have type $\theta$ if they have exact period $n$. But by Theorem 4.2 we need only show that each $J \in S \cup S^{\prime} \cup S^{\prime \prime} \cup S^{\prime \prime \prime}$ is the itinerary of a point of type $\theta$ for some piecewise linear function. But we constructed just such a function in the proof of Lemma 5.2. (The construction is easily modified to include the cases of type $z^{+}, z^{-}$, and $z^{+-}$.)

Conversely, we must show that every sequence of type $\theta$ in $I_{\infty}^{z}, I_{\infty}^{z^{+}}, I_{\infty}^{z^{-}}$, and $I_{\infty}^{z^{+-}}$is in $S, S^{\prime}, S^{\prime \prime}$, and $S^{\prime \prime \prime}$. Suppose $A \in I_{\infty}^{z}$ is of type $\theta$. Let $g$ be the order preserving bijection from the shifts of $A$ to $[n]$. Since $\theta\left(C_{i+1}\right)<_{i} \theta\left(C_{i}\right)$, $S g^{-1}\left(C_{i+1}\right)<_{i} S g^{-1}\left(C_{i}\right)$.

We prove by induction that $g^{-1}\left(C_{i}\right)_{0} \geq L_{i}$. For $i=1$ this is trivial. Assume it is true for some $i \geq 1$. Then $g^{-1}\left(C_{i}\right)_{0} \geq L_{i}$. Hence $g^{-1}\left(C_{i+1}\right)_{0}>$ $g^{-1}\left(C_{i}\right)_{0} \geq L_{i}$. If $g^{-1}\left(C_{i+1}\right)_{0}=L_{i}$ then $L_{i} S\left(g^{-1}\left(C_{i+1}\right)\right)=g^{-1}\left(C_{i+1}\right)>$ $g^{-1}\left(C_{i}\right)=L_{i} S\left(g^{-1}\left(C_{i}\right)\right)$, so $S\left(g^{-1}\left(C_{i+1}\right)\right)>_{i} S\left(g^{-1}\left(C_{i}\right)\right)$, a contradiction. Hence $g^{-1}\left(C_{i+1}\right)_{0} \geq L_{i+1}$. Similarly, by decreasing induction, $g^{-1}\left(C_{i}\right)_{0} \leq L_{i+1}$ for $0 \leq i \leq k+1$.

Now if $C_{i}<j<C_{i+1}$ then

$$
L_{i} \leq g^{-1}\left(C_{i}\right)_{0} \leq g^{-1}(j)_{0} \leq g^{-1}\left(C_{i+1}\right)_{0} \leq L_{i+2} .
$$


If $g^{-1}(j)_{0}=L_{i}$ then as above $S g^{-1}\left(C_{i}\right)<_{i} S g^{-1}(j)$, contradicting $\theta\left(C_{i}\right)>_{i}$ $\theta(j)$. Similarly $g^{-1}(j)_{0}=L_{i+2}$ would contradict $\theta\left(C_{i+1}\right)>_{i+1} \theta(j)$. This shows that $A$ is in $S$.

If $A \in I_{\infty}^{z^{+}}$has type $\theta$, the same argument shows $g^{-1}\left(C_{i}\right)_{0} \geq L_{i}$. Also $g^{-1}(n)_{0} \leq L_{k+2}$. If $g^{-1}(n-1)_{0}=L_{k+2}$ then $n-1<n$ implies $g^{-1}(n-1)<g^{-1}(n)$ which implies $L_{k+2} S g^{-1}(n-1)<L_{k+2} S g^{-1}(n)$ which implies $S g^{-1}(n-1)<_{k} S g^{-1}(n)$, which implies $\theta(n-1)<_{k} \theta(n)$, a contradiction. Thus $g^{-1}(n-1)_{0} \leq L_{k+1}$. The above argument then shows that $A$ is in $S^{\prime}$. The $z^{-}$and $z^{+-}$cases are similar.

\section{A TEST FOR $\theta \rightarrow \pi$ WHEN $z(\theta)=z(\pi)$}

Theorem 6.1. Let $z(\pi)=z$. If $z(\theta)=z, z^{+}, z^{-}$, or $z^{+-}$, then $\theta \rightarrow \pi$ if and only if $F(\theta) \gg F(\pi), F(\theta) \gg i_{+}(F(\pi)), F(\theta) \gg i_{-}(F(\pi))$, or $F(\theta) \gg$ $i_{+} i_{-}(F(\pi))$ respectively.

Proof. If $\theta \rightarrow \pi$ then, by Corollary 4.4, there is a periodic sequence $S \in I_{\infty}^{z(\theta)}$ of type $\pi$ with $F(\theta) \gg A$. By Theorem 5.3, $A \in S$ (or $S^{\prime}, S^{\prime \prime}, S^{\prime \prime \prime}$ ). By Lemma 5.1, $A \gg F(\pi)$ (or $i_{+}(F(\pi))$, etc.).

Conversely, suppose $F(\theta) \gg F(\pi)$ (or $i_{+}(F(\pi)$ ), etc.). If $\pi$ is not a period double then $F(\pi)$ has type $\pi$, by Theorem 5.3, so by Lemma $5.1 \theta \rightarrow \pi$. If $\pi$ is a period double, then we need to show that $F(\theta) \gg F(\pi)$ implies that $F(\theta) \gg J$ for some other $J \in S$ (or $S^{\prime}, S^{\prime \prime}, S^{\prime \prime \prime}$ ). This follows from the following lemma. (Recall that $F(\theta)_{[n-1]}$ is even.)

Lemma 6.2. If $\theta$ and $\pi$ are as in Theorem 6.1, and $F(\theta) \gg F(\pi)$ (or $\left.i_{+}(F(\pi)), i_{-}(F(\pi)), i_{+} i_{-}(F(\pi))\right)$, then there is a $J \in S\left(\right.$ or $\left.S^{\prime}, S^{\prime \prime}, S^{\prime \prime \prime}\right)$ with $J_{[n-1]}$ odd and $F(\theta) \gg J_{[n-1]}$.

The proof of Lemma 6.2 requires an intricate combinatorial theorem, Theorem 7.3, and we postpone it to the end of the next section. First we mention another consequence of Lemma 6.2 and the following lemma.

Lemma 6.3. Let $\theta$ be of type $z, k=|z|$. Then the period doubles of $\theta$ of type $z, z^{+}, z^{-}$, and $z^{+-}$are precisely the permutations $\phi$ of type $z, z^{+}, z^{-}$, and $z^{+-}$for which $F(\phi)$ is an odd sequence of type $\theta$.

Proof. By Theorem 3.3, if $\phi$ is a period double of $\theta$ then $F(\phi)$ is an odd sequence of type $\theta$.

Conversely, suppose that $\phi$ has type $z, z^{+}, z^{-}$, or $z^{+-}$and $F(\phi)$ is an odd sequence of type $\theta$. We will show that in each of the four cases, there are at most $2^{k-1}$ odd sequences of type $\theta$ that could be $F(\phi)$ for some $\phi$, and that $\theta$ has at least $2^{k-1}$ period doubles of each of the four types. Since $F(\phi)$ uniquely determines $\phi$, this would show that $\phi$ is a period double of $\theta$.

Exactly half of the elements of $S, S^{\prime}, S^{\prime \prime}$, and $S^{\prime \prime \prime}$ are odd sequences of type $\theta$. This leaves us with $2^{k-1}, 2^{k}, 2^{k}$, and $2^{k+1}$ such sequences. However not 
all of these can be $F(\phi)$ for some $\phi$. In particular, $F(\phi)$ certainly contains at least one $L_{1}$ and at least one $L_{|z(\phi)|+1}$. But only half of the $2^{k}$ odd sequences of type $\theta$ in $s^{\prime}$ include an $L_{k+2}$ (namely those in $S^{\prime} \backslash i_{+}(S)$ ), and likewise only one half (respectively one fourth) of the $2^{k}\left(2^{k+1}\right)$ elements of $s^{\prime \prime}\left(S^{\prime \prime \prime}\right)$ contain an $L_{1}$ (an $L_{1}$ and an $L_{k+3}$ ). This leaves us with only $2^{k-1}$ possible sequences in each of the four cases.

Now we need only construct $2^{k-1}$ period doubles of $\theta$ of each type. Define $\theta_{*}$ of type $z$ by

$$
\theta_{*}(2 i)= \begin{cases}2 \theta(i) & \text { if } i \in L_{j}(\theta), L_{j} \text { even, } \\ 2 \theta(i)-1 & \text { if } i \in L_{j}(\theta), L_{j} \text { odd, } \\ \text { either } 2 \theta(i) \text { or } 2 \theta(i)-1 & \text { if } i=C_{j}, j \in[k-1], \\ 2 \theta(i)-\delta & \text { if } i=C_{k},\end{cases}
$$

where $\delta$ is 0 or 1 , whichever makes $\theta_{*}(2 i)$ even for an odd number of $i$, and

$$
\theta_{*}(2 i-1)=4 \theta(i)-1-\theta_{*}(2 i) \text {. }
$$

The condition on $C_{k}$ ensures that $\theta_{*}^{n}(1)=2$ (here $n=\operatorname{ord}(\theta)$ ) so $\theta_{*}$ is cyclic. The other conditions ensure that $\theta_{*}$ has the same type as $\theta$. Note that for $i=1, \ldots, k-1$ we have a binary decision about the value of $\theta_{*}(2 i)$. Thus this gives $2^{k-1} \theta_{*}$ 's. If we exchange $\theta_{*}(2 n-1)$ and $\theta_{*}(2 n)\left(\right.$ and $\theta_{*}\left(2 C_{k}\right)$ and $\theta_{*}\left(2 C_{k}-1\right)$ ), we get $2^{k-1} \theta_{*}$ 's of type $z^{+}$. Exchanging $\theta_{*}(1)$ and $\theta_{*}(2)$ gives $2^{k-1}$ of type $z^{-}$, and doing both exchanges gives $2^{k-1}$ of type $z^{+-}$.

Corollary 6.4. Let $z(\pi)=z$ and $z(\theta)=z, z^{+}, z^{-}$, or $z^{+-}$. If $\theta \rightarrow \pi$ and $\theta \neq \pi$ then $\theta \rightarrow \pi_{*}$ for some period double $\pi_{*}$ of $\pi$.

Proof. If $\theta \rightarrow \pi$ then, by Theorem 6.1, $F(\theta) \gg F(\pi)$ (or $i_{+}(F(\pi)$ ), etc.). By Lemma 6.2, $F(\theta) \gg J$ for some odd $J$ of type $\theta$. By Lemma 6.3, $J=F\left(\pi_{*}\right)$ for some period double $\pi_{*}$ of $\pi$. By Theorem 6.1, $\theta \rightarrow \pi_{*}$.

\section{AN INTRICATE COMBINATORIAL THEOREM}

In this section we prove Theorem 7.3, which is required for the proof of Lemma 6.2.

Lemma 7.1. Let $A \in I^{z}$ be a finite odd sequence. If $A^{\infty}$ is $i$-maximal, then it is the $i$-smallest $i$-maximal sequence containing $A$ as a substring immediately following $L_{i}$ or $L_{i+1}$, i.e., if $B$ is $i$-maximal and $B$ contains the substring $L_{i} A$ or $L_{i+1} A$ then $A^{\infty} \leq_{i} B$.

Proof. Suppose otherwise that $B<_{i} A^{\infty}$. Write $B=D A E$, where $D$ ends in $L_{i}$ or $L_{i+1}$. Since $B$ is $i$-maximal, $B=D A E \geq_{i} A E$, so $A E<_{i} A^{\infty}=A A^{\infty}$. Since $A$ is odd, $E>_{i} A^{\infty}>_{i} B$. But the last term of $A$ is $L_{i}$ or $L_{i+1}$ (since $A^{\infty}$ is $i$-maximal), so $E$ is a shift of $B$ following $L_{i}$ or $L_{i+1}$. By $i$-maximality of $B, E \leq_{i} B$, a contradiction. 
Lemma 7.2. Suppose $A$ is even, $A^{\infty}$ is i-maximal, $A^{\prime}$ is $A$ with the last term changed from $L_{i}$ to $L_{i+1}$ or vice versa, and $A^{\prime \infty}$ is i-maximal. Then there is no periodic i-maximal sequence between $A^{\infty}$ and $A^{\prime \infty}$.

Proof. If $L_{i}$ is even, the $L_{i}<_{i} L_{i+1}$, so for $E$ even, $E L_{i}<_{i} E L_{i+1}$, and for $E$ odd, $E L_{i}>_{i} E L_{i+1}$. Thus, whichever of $E L_{i}$ and $E L_{i+1}$ is even is $i$-smaller. If $L_{i}$ is odd, we obtain the same result. Hence $A<_{i} A^{\prime}$.

Let $|A|=n$. Suppose there is an $i$-maximal periodic sequence $B$ with $A^{\infty}<_{i} B<_{i} A^{\prime \infty}$. Since $A_{[n-2]}=A_{[n-2]}^{\prime}$, and $A_{n-1}$ and $A_{n-1}^{\prime}$ are consecutive, we must have $B_{[n-1]}=A$ or $A^{\prime}$. If $B=A^{\prime} C$ then Lemma 7.1 gives $A^{\prime \infty}<_{i} B$, a contradiction. So $B=A C$. Since $B$ is $i$-maximal and $A$ ends in $L_{i}$ or $L_{i+1}, B=A C \geq_{i} C$. Since $A$ is even, prefixing both sides with $A^{m-1}$ does not change the direction of inequality. Thus $A^{m} C \geq_{i} A^{m-1} C$ for all $m>0$. Combining these, we get $A^{m} C \geq_{i} A C=B$ for all $m$. Hence $A^{\infty} \geq_{i} B$, a contradiction.

Let $A_{i_{0}}, A_{i_{1}}, \ldots, A_{i_{k-1}}$ be finite strings and $A=\left(A_{i_{0}} A_{i_{1}} \cdots A_{i_{k-1}}\right)^{\infty} \in I_{\infty}^{z}$ have the property that

$$
\left(A_{i_{r}} A_{i_{r+1}} \cdots A_{i_{k-1}} A_{i_{0}} \cdots A_{i_{r-1}}\right)^{\infty}=A_{\left(i_{r}\right)}
$$

and $A_{i_{r}}$ has the same parity as $i_{r+1}-i_{r}$. Define $A_{i_{j}}^{\prime}=A_{i_{j}}$ except in the last term which is changed from $L_{i_{j+1}}$ to $L_{i_{j+1}+1}$ or vice-versa. Note that a slight modification of the first paragraph of the proof of Lemma 7.2 shows that $A_{i_{j}}<_{i_{j}} A_{i_{j}}^{\prime}$. Define $A_{i_{j}}^{0}=A_{i_{j}}$ and $A_{i_{j}}^{1}=A_{i_{j}}^{\prime}$. If $x$ is a sequence of 0 's and 1's of length $k$, define $I_{x}=\left(A_{i_{0}}^{x_{0}} A_{i_{1}}^{x_{1}} \cdots A_{i_{k-1}}^{x_{k-1}}\right)^{\infty}$. Note that $I_{00 \cdots 0}=A$.

Theorem 7.3. Suppose $z(\pi)=z$ and $A$ and $I_{x}$ are as above. If $F(\pi) \gg A$ then for some $x$ with an odd number of 1 's, $F(\pi) \gg I_{x}$.

The proof of Theorem 7.3 requires three lemmas, which we prove first.

To avoid excess double subscripting, we define $C^{(j)}=C_{\left(i_{j}\right)}, C^{(j+k)}=C^{(j)}$, and we will say $\pi^{(j)}>x$ to mean $\pi^{(j)}>_{i_{j}}\left(A_{i_{j}}^{x_{0}} A_{i_{j+1}}^{x_{1}} \cdots A_{i_{j-1}}^{x_{k-1}}\right)^{\infty}$. Thus we have that $F(\pi) \gg I_{x}$ is equivalent to $\pi^{(j)}>S^{j}(x)$ for all $j$, where $S(x)$ is the circular left shift of $x$, i.e., $S\left(x_{0} x_{1} \cdots x_{k-1}\right)=x_{1} x_{2} \cdots x_{k-1} x_{0}$.

We order the $2^{k}$ sequences of 0 's and 1's of length $k$ as itineraries of the tent map: suppose $x_{i}=y_{i}$ for $i<r$ and $x_{r} \neq y_{r}$; if $x_{0} x_{1} \cdots x_{r-1}$ contains an even number of 1's, then $x<y$ if $x_{r}<y_{r}$, otherwise $x<y$ if $x_{r}>y_{r}$. Note that if $x_{i}=y_{i}$ for $i<r$ and $x_{r} \neq y_{r}$ then $x<y$ if and only if $x_{0} x_{1} \cdots x_{r}$ has an even number of 1's. Also note that $I_{x}<i_{0} I_{y}$ if and only if $x<y$.

Define $x+1$ to be the successor of $x$ in this ordering. Define $x$ to be even (odd) if it contains an even (odd) number of 1's. We note a few facts about successors: $x$ and $x+1$ disagree in exactly 1 term; if $x$ is even then it differs 
from $x+1$ only in the last term; if $x$ is odd and $x_{k-1}$ is a 1, then $x$ and $x+1$ disagree only in the next to last term, $x_{k-2}$.

Lemma 7.4. If $x$ is even and $\pi^{(j)}>x$, then $\pi^{(j)}>x+1$.

Proof. Since $x$ is even, $x+1$ differs from $x$ only in the last term. Note that $\left(A_{i_{0}}^{x_{0}} \cdots A_{i_{k-1}}^{x_{k-1}}\right)$ is even if and only if $x$ is even. The lemma then just reduces to Lemma 7.2.

Theorem 7.5. If the first $r$ terms of $x$ and $y$ agree and $x<\pi^{(j)}<y$ then $\min \left(S^{r+1}(x), S^{r+1}(y)\right)<\pi^{(j+r+1)}$.

Proof. Rephrasing the assumption, we have

$$
\begin{aligned}
A_{i_{j}}^{x_{0}} A_{i_{j+1}}^{x_{1}} \cdots A_{i_{j+r}}^{x_{r}} \cdots & <_{i_{j}} \pi_{\left(i_{j}\right)}<_{i_{j}} A_{i_{j}}^{y_{0}} A_{i_{j+1}}^{y_{1}} \cdots A_{i_{j+r-1}}^{y_{r-1}} A_{i_{j+r}}^{y_{r}} \cdots \\
& =A_{i_{j}}^{x_{0}} A_{i_{j+1}}^{x_{1}} \cdots A_{i_{j+r-1}}^{x_{r-1}} A_{i_{j+r}}^{y_{r}} \cdots .
\end{aligned}
$$

Since $A_{i_{j+r}}^{x_{r}}$ and $A_{i_{j+r}}^{y_{r}}$ agree in all except possibly the last term, and their last terms are consecutive (or equal), $\pi_{\left(i_{j}\right)}=A_{i_{j}}^{x_{0}} A_{i_{j+1}}^{x_{1}} \cdots A_{i_{j+r-1}}^{x_{r-1}} A_{i_{j+r}}^{x_{r}} B$ or $A_{i_{j}}^{x_{0}} A_{i_{j+1}}^{x_{1}} \cdots$ $A_{i_{j+r-1}}^{x_{r-1}} A_{i_{j+r}}^{y_{r}} B$. We consider the two cases $x_{r}=y_{r}$ and $x_{r} \neq y_{r}$.

Suppose $x_{r} \neq y_{r}$. Then $x<y$ implies $x_{0} \cdots x_{r}$ is even. Hence $A_{i_{j}}^{x_{0}} A_{i_{j+1}}^{x_{1}} \ldots$ $A_{i_{j+r}}^{x_{r}}$ has the same parity as $A_{i_{j}} A_{i_{j+1}} \cdots A_{i_{j+r}}$ which has the same parity as $i_{j+r+1}-i_{j}$. Suppose $\pi_{\left(i_{j}\right)}=\cdots A_{i_{j+r}}^{x_{r}} B$. Thus $A_{i_{j}}^{x_{0}} A_{i_{j+1}}^{x_{1}} \cdots A_{i_{j+r}}^{x_{r}} A_{i_{j+r+1}}^{x_{r+1}} \cdots<_{i_{j}}$ $A_{i_{j}}^{x_{0}} A_{i_{j+1}}^{x_{1}} \cdots A_{i_{j+r}}^{x_{r}} B$ implies $A_{i_{j+r+1}}^{x_{r+1}}<_{i_{j+r+1}} B$. Since $B$ follows $A_{i_{j+r}}^{x_{r}}$ which ends in $L_{i_{j+r+1}}$ or $L_{i_{j+r+1}+1}, B \leq_{i_{j+r+1}} \pi_{\left(i_{j+r+1}\right)}$, so $S^{r+1}(x)<\pi^{(j+r+1)}$. On the other hand, if $\pi_{\left(i_{j}\right)}=\cdots A_{i_{j+r}}^{y_{r}} B$ then since $A_{i_{j}}^{y_{0}} \cdots A_{i_{j+r}}^{y_{r}}$ has the opposite parity of $i_{j+r-1}-i_{j}, B>_{i_{j+r+1}} A_{i_{j+r+1}^{y_{r+1}} \ldots} \ldots$ so $S^{r+1}(y)<\pi^{(j+r+1)}$.

Suppose instead that $x_{r}=y_{r}$. If $x_{0} x_{1} \cdots x_{r}$ is even then

$$
A_{i_{j}}^{x_{0}} \cdots A_{i_{j+r}}^{x_{r}} A_{i_{j+r+1}}^{x_{r+1}} \cdots<_{i_{j}} A_{i_{j}}^{x_{0}} \cdots A_{i_{j+r}}^{x_{r}} B
$$

implies $S^{r+1}(x)<\pi^{(j+r+1)}$, otherwise $A_{i_{j}}^{y_{0}} \cdots A_{i_{j+r}}^{y_{r}} B<_{i_{j}} A_{i_{j}}^{y_{0}} \cdots$ implies $S^{r+1}(y)$ $<\pi^{(j+r+1)}$.

In all cases $\min \left(S^{r+1}(x), S^{r+1}(y)\right)<\pi^{(j+r+1)}$.

Theorem 7.6. Let $x$ be odd and suppose that $x$ and $x+1$ agree to exactly $r$ terms. If for some $m, \min \left(S^{i}(x), S^{i}(x+1)\right)<\pi^{(m+i)}$ for $i=0, \ldots, r+1$, then for some odd $y, S^{i}(y)<\pi^{(i)}$ for all $i$.

Proof. We proceed by induction on $x$. For $x=00 \cdots 001, x+1=00 \cdots 011$. Then $S^{i}(x)<S^{i}(x+1)$ for $i=0,1, \ldots, k-2$. Since $x$ and $x+1$ agree to $i$ terms for each $i \leq k-2$, by Lemma $7.5 S^{i}(x)=\min \left(S^{i}(x), S^{i}(x+1)\right)<\pi^{(m+i)}$ for $0 \leq i \leq k-2$ and $100 \cdots 01=S^{k-1}(x+1)=\min \left(S^{k-1}(x), S^{k-1}(x+1)\right)<$ $\pi^{(m+k-1)}$. Since the latter is even, $\pi^{(m+k-1)}>S^{k-1}(x+1)+1=S^{k-1}(x)$, 
so $S^{i}(x)<\pi^{(m+i)}$ for all $i$. Hence if we let $y=S^{k-m}(x)$ then $S^{i}(y)=$ $S^{k-m+i}(x)<\pi^{(m+k-m+i)}=\pi^{(k+i)}=\pi^{(i)}$ for all $i$.

Now suppose $x$ is odd and the result has been shown for all smaller odd $x$. Suppose that $\pi^{(l)}<x$ for some $l$. Then $\pi^{(l)}<x-1$. So for some odd $z<x$, $z<\pi^{(l)}<z+1$. By Lemma 7.5, if $z$ and $z+1$ agree to exactly $r$ terms then $\min \left(S^{i}(z), S^{i}(z+1)\right)<\pi^{(l+i)}$ for $0 \leq i \leq r+1$. Thus by our inductive hypothesis we are done. So we may assume that $x<\pi^{(l)}$ for all $l$.

We consider two cases.

First suppose that $x$ ends in a 0 . If we let $z=S^{-1}(x)$ then $z<x$ and $z$ is odd. If $z$ and $z+1$ agree to exactly $p$ terms then $x$ and $x+1$ agree to exactly $p-1$ terms. (Except in the case that $z$ and $z+1$ agree to 0 terms, in which case $z=010 \cdots 0, z+1=110 \cdots 0, x=100 \cdots 0$, and $x+1$ is not even defined so the theorem is vacuously true.) By assumption $\min \left(S^{i}(x) S^{i}(x+1)\right)<\pi^{(m+i)}$ for $0 \leq i \leq p$. If $\pi^{(m-1)}<z$ then $\pi^{(m-1)}<x$ and we are done, so we may assume $\pi^{(m-1)}>z$. Then $\min \left(S^{i}(z), S^{i}(z+1)\right)<\pi^{(m-1+i)}$ for $0 \leq i \leq p+1$, so by our inductive hypothesis we are done.

Next suppose that $x$ ends in a 1 . Then $x$ and $x+1$ agree to exactly $k-2$ terms. By assumption $\min \left(S^{i}(x), S^{i}(x+1)\right)<\pi^{(m+i)}$ for $0 \leq i \leq k-1$. If $S^{i}(x)<\pi^{(m+i)}$ for $0 \leq i \leq k-1$ then we are done, so we may assume that $S^{i}(x+1)<\pi^{(m+i)}<S^{i}(x)$ for some $i$. Choose $i$ smallest with this property. By Lemma 7.4, $S^{i}(x+1)+1<\pi^{(m+i)}<S^{i}(x)-1$. Let $z=S^{-1}\left(S^{i}(x+1)+1\right)$. Let $u=x_{0} x_{1} \cdots x_{i-1}, v=x_{i} \cdots x_{k-1}$. Note that since $S^{k-1}(x)$ and $S^{k-1}(x+1)$ disagree in only the last term, they ae consecutive, so by Lemma $7.4 \pi^{(m+k-1)}$ cannot be between them; thus $i<k-1$. Define $v^{\prime \prime}=v$ with the next to last term changed. Then $x=u v, x+1=u v^{\prime \prime}, S^{i}(x)=v u, S^{i}(x+1)=v^{\prime \prime} u$. Since we are assuming $S^{i}(x+1)<S^{i}(x)$, we have $v^{\prime \prime}<v$. But since $x<x+1$, we must have $u v<u v^{\prime \prime}$. Hence $u$ is odd. Define $u^{\prime}=u$ with the last term changed. Since $S^{i}(x+1)=v^{\prime \prime} u$ is even, its successor differs from it only in the last term. Thus $S^{i}(x+1)+1=v^{\prime \prime} u^{\prime}$. Hence $z=u^{\prime} v^{\prime \prime}$. Since $u$ is odd, $u^{\prime}<u$. Thus $z<x$. Also $z$ is odd, and the last digit of $z$ is the last digit of $x$ which is a 1 . Thus $z+1$ differs from $z$ only in the next to last digit, i.e., $z+1=u^{\prime} v$. So $S^{i}(z+1)=v u^{\prime}=v u-1=S^{i}(x)-1$.

Since by assumptions $S^{i}(x+1)+1=S^{i}(z)<\pi^{(m+i)}<S^{i}(z+1)=S^{l}(x)-1$, and $S^{i}(z)$ and $S^{i}(z+1)$ agree to $k-2-i$ terms, $\min \left(S^{j}(z), S^{j}(z+1)\right)<$ $\pi^{(m+j)}$ for $i \leq j \leq k-1$. If this were true for $0 \leq j \leq k-1$ then by our inductive hypothesis we would be done. If not then for some $j$ with $0<j<i$, $\min \left(S^{j}(z), S^{j}(z+1)\right)>\pi^{(m+j)}$. Since $j<i$ and $i$ was chosen smallest with the property $S^{i}(x)>\pi^{(m+i)}$, we must have

$$
S^{j}(x)<\pi^{(m+j)}<\min \left(S^{j}(z), S^{j}(z+1)\right) \leq S^{j}(z+1) .
$$

But since $S^{i}(z+1)+1=S^{i}(x)$ and $S^{i}(z+1)$ is even, $S^{i}(z+1)$ and $S^{i}(x)$ differ only in the last term. Thus $S^{j}(x)$ and $S^{j}(z+1)$ differ only in the $(i-j)$ th 
term. Thus by Lemma 7.5

$$
\begin{aligned}
\pi^{(m+i)} & >\min \left(S^{i-j}\left(S^{j}(x)\right), S^{i-j}\left(S^{j}(z+1)\right)\right) \\
& =\min \left(S^{i}(x), S^{i}(z+1)\right)=S^{i}(z+1)=S^{i}(x)-1 .
\end{aligned}
$$

This contradicts our assumption that $\pi^{(m+i)}<S^{i}(x)-1$.

Proof of Theorem 7.3. If $\pi^{(m)}>x$ for all $m$ and all odd $x$ the result is obvious. Otherwise for some $m$ and odd $x, x<\pi^{(m)}<x+1$. Then by Lemma $7.5 \min \left(S^{i}(x), S^{i}(x+1)\right)<\pi^{(m+i)}$ for $0 \leq i \leq r+1$, where $x$ and $y$ agree to exactly $r$ terms. The theorem then follows from Lemma 7.6.

Proof of Lemma 6.2. Let $i_{+} i_{-}\left(I_{\theta}^{\prime \prime \prime}(1)\right)=\left(R_{1} C_{i_{1}} R_{2} C_{i_{2}} \cdots R_{k+2} C_{i_{k+2}}\right)^{\infty}$, where $R_{1}, R_{2}, \ldots$ contains no $C$ 's and $i_{1}, \ldots, i_{k+2}$ is a permutation of $[k+2]$. (If 1 is not $\theta\left(C_{i}\right)$ for any $i$ then change that to $i_{+} i_{-}\left(I_{\theta}^{\prime \prime \prime}\left(\theta\left(C_{1}\right)\right)\right)$.) Define for $1 \leq r \leq k+2, A_{i_{r-1}}=R_{r} L_{i_{r}}$ or $R_{r} L_{i_{r+1}}$, whichever gives $A_{i_{r}}$ the same parity as $i_{r+1}-i_{r}$. Then the sequences $I_{x}$ are shifts of the sequences of $S^{\prime \prime \prime}$, and the minimal one, $I_{00 \ldots 0}$, is $i_{+} i_{-} F(\pi)$. Thus the $S^{\prime \prime \prime}$ case of the lemma follows from Theorem 7.3. The cases of $S, S^{\prime}$, and $S^{\prime \prime}$ follow similarly.

\section{NONEXISTENCE OF MINIMAL UPPER BOUNDS}

Theorem 8.1. If $\theta$ is not a period double, then there is a sequence of distinct cyclic permutations $\theta_{h}$ such that:

(i) $\theta \rightarrow \theta_{n}$ for all $h$.

(ii) if $\pi \rightarrow \theta_{h}$ for all $h$ then $\pi \rightarrow \theta$.

(iii) If $\theta \rightarrow \pi \neq \theta$ then $\theta_{h} \rightarrow \pi$ for all sufficiently large $h$.

Proof. Bernhardt has constructed a sequence having the first two properties [2, Lemma 4.4]. (He calls them $\theta_{h n+k}$ ) The construction can be summarized as follows. Let $n=\operatorname{ord}(\theta)$. Since $\theta^{n}(1)=1$ and $\theta^{k}(2)=1$ for some $k<n$, for any $h \geq 1$ there is a periodic orbit of period $h n+k$ corresponding to the loop in the Markov graph found by taking a point slightly greater than 1 that follows the orbit of 1 for $h n$ steps and then follows the orbit of 2 for $k$ steps. Let $x$ be the start point of this orbit and let $\theta_{h}$ be the type of $x$. We will show that $\theta_{h}$ has the third property.

Let $F(\theta)=A^{\infty},|A|=\operatorname{ord}(\theta)$. Clearly $A^{h}$ is a prefix of the itinerary of $x$. Since this itinerary differs from $F\left(\theta_{h}\right)$ in at most $z(\theta)$ terms, for all $m$ and for $h$ sufficiently large (depending on $m$ ), $F\left(\theta_{h}\right)$ contains $A^{m}$ as a substring. Hence $F\left(\theta_{h}\right)$ has a shift following $L_{i}$ or $L_{i+1}$ which agrees with $\theta_{(i)}$ to any desired number of terms.

Suppose $\theta \rightarrow \pi \neq \theta$. Then there is a sequence $B$ in $I_{\infty}^{z(\theta)}$ of type $\pi$ with $F(\theta) \gg B$. For $h$ sufficiently large, $\left(\theta_{h}\right)_{(i)}$ is at least as large (with respect to $>_{i}$ ) as each of its shifts which follow $L_{i}$ or $L_{i+1}$, which include a sequence that agrees with $\theta_{(i)}$ to more terms than does $B_{(i)}$. Hence $\left(\theta_{h}\right)_{(i)}>_{i} B_{(i)}$. Since 
there are only finitely many values of $i$ under consideration, we can choose $h$ large enough that $\left(\theta_{h}\right)_{(i)}>_{i} B_{(i)}$ for all $i$. Hence $\theta_{h} \rightarrow \pi$.

Theorem 8.2. If $\theta \nrightarrow \pi$ and $\pi \nrightarrow \theta$ then $\pi$ and $\theta$ have no minimal upper bound in the partial ordering $\rightarrow$.

Proof. Suppose to the contrary that $\phi \rightarrow \pi, \phi \rightarrow \theta$, and $\phi$ is $\rightarrow$-minimal with this property. The assumptions on $\pi$ and $\theta$ imply that $\phi \neq \pi$ and $\phi \neq \theta$. If $\phi$ were a period double of $\phi_{*}$ then, by the remark in $\S 3, \phi$ is an immediate successor of $\phi_{*}$, so $\phi \rightarrow \phi_{*}, \phi_{*} \rightarrow \pi$, and $\phi_{*} \rightarrow \theta$. Thus $\phi$ is not a minimal upper bound. If $\phi$ is not a period double then for $h$ sufficiently large, the $\phi_{h}$ constructed in the above theorem satisfies $\phi \rightarrow \phi_{h}, \phi_{h} \rightarrow \pi$, and $\phi_{h} \rightarrow \theta$, so again $\phi$ is not minimal.

\section{MAXimal elements of $C^{n}$}

In this section we characterize the maximal elements of $\rightarrow$ restricted to $C^{n}$ for fixed $n$. We first prove that for fixed $n$ and $z$, all maximal elements of $\{\theta: \operatorname{ord}(\theta)=n, z(\theta)=z\}$ must have a certain form. Essentially, they must have the property that all noncritical points have the smallest or largest possible itinerary. Next we prove a conjecture of Baldwin [1, Conjecture 6.3] that maximal elements of $C^{n}$ must have type $\pm(n-2)$. Finally, we give necessary and sufficient conditions for permutations of type $\pm(n-2)$ to be maximal elements of $C^{n}$.

Define $P(z)=\{\theta: z(\theta)=z\}$. Given $\theta \in C^{n}, z(\theta)=z, k=|z|$, let $m$ and $M$ be the minimum and maximum sequence in $I_{\infty}^{z}$, e.g., if $L_{1}$ is even then $m=L_{1} L_{1} \ldots$ Let $I_{\theta}\left(C_{1}\right)=I_{\theta}\left(C_{i_{1}}\right)=\left(C_{i_{1}} A_{1} C_{i_{2}} A_{2} \cdots C_{i_{k}} A_{k}\right)^{\infty}$, where each $A_{i}$ (which could be empty) contains only $L$ 's. Let $B(\theta)=B=$ $\left(C_{i_{1}} B_{1} C_{i_{2}} B_{2} \cdots C_{i_{k}} B_{k}\right)^{\infty}$, where $\left|B_{j}\right|=\left|A_{j}\right|$ and $B_{j}$ is a prefix of $m$ if $C_{i_{j}}$ is a minimum and of $M$ if $C_{i_{j}}$ is a maximum. Let $g$ be the u.o.p.b. from $\left\{B, S(B), \ldots, S^{n-1}(B)\right\}$ to $[n]$, and $\pi=g \circ S \circ g^{-1}$.

In what follows, we will use $C_{i}$ to mean three things: $C_{i}(\theta), C_{i}(\pi)$, and $C_{i} \in$ $I^{z}$. In most cases the context should suggest the meaning, for example $g^{-1}\left(C_{i}\right)$ means $g^{-1}\left(C_{i}(\pi)\right), I_{\theta}\left(C_{i}\right)$ means $I_{\theta}\left(C_{i}(\theta)\right)$, and $\theta\left(C_{i}\right)$ means $\theta\left(C_{i}(\theta)\right)$. We will also use $C_{i} \cdots$ to mean the shift of $B$ that begins with $C_{i}$.

Lemma 9.1. If $I_{\theta}\left(C_{1}\right) \neq B(\theta)$ then $z(\pi)=z(\theta), \pi \neq \theta$, and $\pi \rightarrow \theta$.

Corollary 9.2. If $\theta$ is a maximal element of $C^{n} \cap P(z)$ then $I_{\theta}\left(C_{1}\right)=B(\theta)$.

Proof of Lemma 9.1. Since in forming $B$ we have made the maxima of $\theta$ "as high as possible" and the minima "as low as possible", it should be easy to show that $\pi \rightarrow \theta$. However, $I_{\pi}\left(C_{1}\right)$ is not necessarily $B$, and this complicates matters greatly.

We begin by finding the critical points of $\pi$. Let $p=g(B)$. Then clearly $g\left(C_{i} \cdots\right)=p+i-1$, since no shifts of $B$ lies between $\left(C_{j} \cdots\right)$ and $\left(C_{j+1} \cdots\right)$. 
First we show that for $0 \leq j<k-1, \pi(p+j)>_{j+1} \pi(p+j+1)$. (Here $<_{j+1}$ is with respect to $z$.) Let $g^{-1}(p+j)=S^{r}(B), g^{-1}(p+j+1)=S^{S}(B)$. We wish to show that $S^{r+1}(B)>_{j+1} S^{s+1}(B)$. Assume $C_{j+1}$ is a maximum, the other case being similar. Since $(B)_{r}=C_{j+1},(B)_{r+1}=L_{k+1}$ or $C_{l}$ for some $l$. Similarly $(B)_{s+1}=L_{1}$ or $C_{m}$. The only case for which $S^{r+1}(B)>$ $S^{s+1}(B)$ is not obvious is $(B)_{r+1}=C_{l},(B)_{s+1}=C_{m}$. But then $I_{\theta}\left(C_{1}\right)_{r}=$ $C_{j+1}, I_{\theta}\left(C_{1}\right)_{r+1}=C_{l}, I_{\theta}\left(C_{1}\right)_{s}=C_{j+2}, I_{\theta}\left(C_{1}\right)_{s+1}=C_{m}$, so $\theta\left(C_{j+1}\right)=C_{l}$ and $\theta\left(C_{j+2}\right)=C_{m}$. But we know that $\theta\left(C_{j+1}\right)>\theta\left(C_{j+2}\right)$ since $C_{j+1}$ is a maximum, so $C_{l}>C_{m}$. Hence $S^{r+1}(B)>_{j+1} S^{s+1}(B)$.

Next we show that for $1 \leq j<p-1, \pi(j)<_{1} \pi(j+1)$. Let $g^{-1}(j)=S^{r}(B)$, $g^{-1}(j+1)=S^{S}(B)$. Then since $g^{-1}$ is order preserving, $S^{r}(B), S^{S}(B)<$ $B=g^{-1}(p)=C_{1} \ldots$, so $(B)_{r}=(B)_{s}=L_{1}$. Since $L_{1} S^{r+1}(B)=S^{r}(B)<$ $S^{S}(B)=L_{1} S^{S+1}(B)$, and hence $S^{r+1}(B)<_{1} S^{S+1}(B)$, we have $\pi(j)<_{1} \pi(j+1)$. Similarly, for $j \geq p+k, \pi(j)<_{k+1} \pi(j+1)$.

Now we show that $\pi(1)<_{1} \pi(2)$. This follows from the above results unless $p=1$ or 2 . Since 1 is the global minimum of $\theta, \theta^{-1}(1)=n$ or $C_{i}$ for some $i$. If $\theta\left(C_{i}\right)=1$, then $C_{i}$ is followed by an $L_{1}$ in $I_{\theta}\left(C_{1}\right)$, hence in $B$. Since $B$ contains an $L_{1}, p \geq 2$. If $\theta(n)=1$, then $\theta^{-1}(n)=C_{i}$ for some $i$, since $\theta^{-1}(n)=1$ would imply that $\theta^{2}(1)=1$, a contradiction. Hence $I_{\theta}\left(C_{1}\right)$ contains the substring $C_{i} L_{k+1} L_{1}$. Also, $n$ is a local minimum, so $L_{k+1}$ is odd, hence $M=L_{k+1} L_{1} \ldots$. Thus $B$ also contains this substring, and again $p \geq 2$.

If $p=2$, then $B$ contains exactly one $L_{1}$ in each substring of length $n$. The string in $B$ following this $L_{1}$ is $g^{-1}(\pi(1))$, and begins with some $C_{j}$ or with $L_{1}$ (respectively $L_{k+1}$ ) if $L_{1}$ is even (respectively odd). Since $C_{1}$ is a maximum or minimum if $L_{1}$ is even or odd, respectively, $g^{-1}(\pi(p))$ starts with some $C_{l}$ or with $L_{k+1}$ (respectively $\left.L_{1}\right)$ if $L_{1}$ is even (respectively odd). The only case for which it is not clear that $\pi(1)<_{1} \pi(2)$ is $g^{-1}(\pi(1))_{0}=C_{j}$, $g^{-1}(\pi(2))_{0}=C_{l}$. But then $\theta(1)=C_{j}, \theta\left(C_{1}\right)=C_{l}$, and we already know that $\theta(1)<_{1} \theta\left(C_{1}\right)$ so we are done. Similarly, $\theta(n)<_{k} \theta(n-1)$.

In summary, as a consequence of what we have shown, $z(\pi)=z(\theta), C_{i}(\pi)=$ $p+i-1$ for $2 \leq i \leq k-1, C_{1}(\pi)=p$ or $p-1$, and $C_{k}(\pi)=p+k-1$ or $p+k$.

The proof that $\pi \rightarrow \theta$ is fairly simple if $C_{1}(\pi)=p$ and $C_{k}(\pi)=p+k-1$ but becomes tedious in the other cases. Recall that we need only show that $\pi_{(i)}>_{i} \theta_{(i)}$ for all $i$.

First we show that if $C_{1}(\pi)=p-1$ then $\theta\left(C_{1}(\theta)\right)=C_{a}(\theta)$ for some $a$, and $g^{-1}\left(C_{1}(\pi)\right)_{1}=C_{b}$ for some $b$. Define $L_{\max }=L_{k+1}$ if $L_{1}$ is even and $L_{1}$ if $L_{1}$ is odd, and define $L_{\min }$ to be whichever of $L_{1}$ and $L_{k+1}$ is not $L_{\max }$. Since $C_{1}(\pi)=p-1, g^{-1}\left(C_{1}(\pi)\right)_{0}=L_{1}$. In both $m$ and $M$ the term following an $L_{1}$ must be an $L_{\min }$. Thus $g^{-1}\left(C_{1}(\pi)\right)_{1}=L_{\min }$ or $C_{b}$ for some $b$. Recall 
that $C_{1}=(B)_{0}$. Then $(B)_{1}=L_{\max }$ or $C_{a}$ for some $a$. If $C_{1}(\pi)=p-1$ then $\pi(p-1)>_{1} \pi(p), g^{-1}(\pi(p-1))>_{1} g^{-1}(\pi(p)), S\left(g^{-1}(p-1)\right)>_{1} S\left(g^{-1}(p)\right)$, $g^{-1}(p-1)_{1} \geq_{1} g^{-1}(p)_{1}$, so $L_{\min }$ or $C_{b} \geq_{1} L_{\max }$ or $C_{a}$. The only way that this can happen is if $g^{-1}\left(C_{1}(\pi)\right)_{1}=C_{b}$ and $(B)_{1}=C_{a}$ so $\theta\left(C_{1}\right)=C_{a}$ and $C_{b} \geq_{1} C_{a}$. They are unequal since $\theta$ is one-to-one. Since $g^{-1}\left(C_{1}(\pi)\right)_{1}=C_{b}$, $\pi\left(C_{1}\right)=g\left(C_{b} \cdots\right)=C_{b}$ (except possibly if $b=k$ or 1 and $\pi\left(C_{1}\right) \in L_{k}$ or $L_{2}$ ). In all cases $\pi_{(1)}>_{1} \theta_{(1)}$. (If $b=k, \pi\left(C_{1}\right) \in L_{k}$, and $a=k-1$ then $C_{b} \geq_{1} C_{a}$ implies that $L_{1}$ is even, so $C_{1}$ is a maximum, so points near $C_{1}(\theta)$ go under $f_{\theta}$ to points less than $C_{k-1}$, so $\theta_{(1)}=S\left(I_{\theta}^{+}\left(C_{1}\right)\right)=L_{k-1} \cdots<_{1} L_{k} \cdots=\pi_{(1)}$. The $b=1$ case is similar.)

The proof that $\theta_{(k)}<{ }_{k} \pi_{(k)}$ if $C_{k}(\pi)=p+k$ is similar. For future reference, let $C_{c}$ and $C_{d}$ play the roles of $C_{a}$ and $C_{b}$ respectively.

This shows that if $C_{1}(\pi)=p-1$ or $C_{k}(\pi)=p+k$ then $\theta_{(1)} \neq \pi_{(1)}$ or $\theta_{(k)} \neq \pi_{(k)}$. On the other hand, if $C_{1}(\pi)=p$ and $C_{k}(\pi)=p+k-1$ then $I_{\pi}\left(C_{1}(\pi)\right)=B$ which by assumption is not equal to $I_{\theta}\left(C_{1}(\theta)\right)$. Either way $\theta \neq \pi$.

In the case that $C_{1}(\pi)=p-1$, let $x=\theta^{-1}\left(C_{b}\right)$. Since $\theta(x)=C_{b}>_{1} C_{a}=$ $\theta\left(C_{1}\right)>_{1} \theta(t)$ for all $t \in L_{1} \cup L_{2}$, it follows that $x \notin L_{1} \cup L_{2}$. Since the term in $B$ preceding $C_{b}$ is an $L_{1}, \theta^{-1}\left(C_{b}\right) \neq C_{r}$ for any $r$. Thus $x>C_{2}(\theta)$. Similarly, if $y=\theta^{-1}\left(C_{d}\right)$ in the case $C_{k}(\pi)=p+k$ then $y<C_{k-1}(\theta)$.

Assume $\pi_{(i)}<_{i} \theta_{(i)}$ for some $i$. Assume that $i$ is chosen so that $\pi_{(i)}<_{i} \theta_{(i)}$ and so as to make the smallest $r$ for which $\pi_{(i) r} \neq \theta_{(i) r}$ as small as possible. We know that if $C_{1}(\pi)=p-1$ then $i \neq 1$ and if $C_{k}(\pi)=p+k$ then $i \neq k$, so $g^{-1}\left(C_{i}(\pi)\right)_{0}=C_{i}$. Let $S\left(I_{\theta}\left(C_{i}\right)\right)=U, S\left(I_{\pi}\left(C_{i}\right)\right)=V, S\left(g^{-1}\left(C_{i}(\pi)\right)\right)=W$. We know that $V$ and $W$ agree at least until one of them is $C_{1}$ or $C_{k}$ and $U$ and $W$ agree in all terms in which either is a $C_{j}$. Suppose that $U_{[m-1]}=$ $V_{[m-1]}, U_{m} \neq V_{m}$.

Suppose $U_{[m-1]}$ contains some $C_{j}$, say $U_{l}=C_{j}$. Then $V_{l}=C_{j}$. Thus $\pi_{(j)}$ and $\theta_{(j)}$ agree to fewer terms than $\pi_{(i)}$ and $\theta_{(i)}$. By our choice of $i$, $\pi_{(j)}>_{j} \theta_{(j)}$. We know that $\pi_{(i)[l]}$ is even if and only if $i$ and $j$ have the same parity. Thus $U_{[m-1]}$ contains no $C_{j}$. Hence $W_{[m-1]}$ contains no $C_{j}$. Hence $V_{[m-1]}=W_{[m-1]}=m_{[m-1]}$ or $M_{[m-1]}$, whichever is bigger with respect to $>_{i}$.

If $V_{m}$ or $U_{m}$ is $C_{j}$ with $j \neq 1$ and $j \neq k$, then the other must be, so $V_{m}=U_{m}$, contrary to assumption. If $V_{m}=L_{u}$ and $U_{m}=L_{v}$ then $V_{[m]}=$ $W_{[m]}=\pi_{(i)[m]}=$ the biggest sequence of $m+1$ terms with respect to $>_{i}$, and $\theta_{(i) m}=L_{v} \neq L_{u}=\pi_{(i) m}$, so $\pi_{(i)}>_{i} \theta_{(i)}$. The only remaining possibility is that $V_{m}$ or $U_{m}$ is $C_{1}$ or $C_{k}$, and the other is not.

We are left with four cases.

Suppose $V_{m}=C_{1}$. Then $W_{m}=L_{1}, W_{m+1}=U_{m+1}=C_{b}$, and $x=$ $\theta^{-1}\left(C_{b}\right)>C_{2}$, so $U_{m}=L_{j}, j>2$. Since $V_{m}=C_{1}, \pi_{(i) m}=L_{1}$ or $L_{2}<U_{m}=\theta_{(i) m}$ and $\pi_{(i)[m-1]}=\theta_{(i)[m-1]}$. Since $W_{m}=L_{1}, L_{e}<L_{f}$ implies $W_{[m-1]} L_{e}>_{i} W_{[m-1]}=\theta_{(i)[m-1]}$. Since $W_{m}=L_{1}, L_{e}<L_{f}$ implies 
$W_{[m-1]} L_{e}>_{i} W_{[m-1]} L_{f}$, so $\pi_{(i)[m]}>_{i} \theta_{(i)[m]}$. Hence $\pi_{(i)}>_{i} \theta_{(i)}$. The case $V_{m}=C_{k}$ is similar.

Now if $U_{m}=C_{1}, V_{m}=L_{2}, W_{m}=C_{1}$, then $U_{m+1}=V_{m+1}=W_{m+1}=C_{a}$, or $U_{m+1}=W_{m+1}=C_{k}, V_{m+1}=L_{k}$. Consider the case that $L_{i}$ is even. The other case is similar. If $U_{[m-1]}$ is even then a point starting near $C_{i}(\theta)$ will, under $f_{\theta}^{m+1}$, end up a little less than $C_{1}$, so $\theta_{(i) m}=L_{1}<L_{2}=\pi_{(i) m}$, so $U_{[m-1]} \theta_{(i) m}<U_{[m-1]} \pi_{(i) m}$, so $\theta_{(i)}<\pi_{(i)}$, and since we assumed that $L_{i}$ is even, $\theta_{(i)}<_{i} \pi_{(i)}$. If $U_{[m-1]}$ is odd, then $\theta_{(i) m}=L_{2}$, so $\theta_{(i)[m]}=\pi_{(i)[m]}$. If $U_{m+1}=V_{m+1}=C_{a}$ then $\theta_{(i)[m+1]}=\pi_{(i)[m+1]}$ and both have the same parity as $a-i$. Again, our choice of $i$ implies that $\theta_{(a)}<_{a} \pi_{(a)}$, so $\theta_{(i)[m+1]} \theta_{(a)}<_{i}$ $\theta_{(i)[m+1]} \pi_{(a)}, \theta_{(i)}<_{i} \pi_{(i)}$. If otherwise $U_{m+1}=C_{k}$ and $V_{m+1}=L_{k}$ then $a=k$, and since $C_{a}<{ }_{1} C_{b}$, this can only happen if $L_{1}$ is odd. Hence $L_{2}$ is even, so $U_{[m]}=U_{[m-1]} L_{2}$ is odd. Hence $\theta_{(i) m+1}=L_{k+1}>L_{k}=\pi_{(i) m+1}$ and $\theta_{(i)}<\pi_{(i)}$. Since we have assumed that $L_{i}$ is even, $\theta_{(i)}<\pi_{(i)}$. The case $U_{m}=C_{k}$, $V_{m}=L_{k}$ is similar.

Lemma 9.3. Suppose $\theta$ is a maximal element of $C^{n} \cap P(z), 2 \in L_{1}(\theta)$, and $\left|\theta^{-1}(1)-\theta^{-1}(2)\right|>1$. Then there is $a \pi \in C^{n} \cap P\left(z^{-}\right)$with $\pi \rightarrow \theta$.

Proof. Let $\pi=(12) \theta(12)$. (Here (12) is the cyclic permutation of 1 and 2.) We will show that $\pi \rightarrow \theta$ and $z(\pi)=z^{-}$. We know that $\theta^{-1}(1) \neq 1$ since 1 has order $n$, and $\theta^{-1}(1) \neq 2$ since if it were then 2 would be a local minimum, contradicting $2 \in L_{1}(\theta)$. Let $\theta^{-1}(1)=p, \theta^{-1}(2)=q$. Then

$$
\pi(i)= \begin{cases}\theta(2) & \text { if } i=1, \\ \theta(1) & \text { if } i=2, q \neq 1 \\ \theta(1)-1 & \text { if } i=2, \quad q=1 \\ \theta(p)+1 & \text { if } i=p \\ \theta(q)-1 & \text { if } i=q \neq 1 \\ \theta(i) & \text { otherwise. }\end{cases}
$$

Since in forming $\pi$ from $\theta$ we change the value at each point other than 1 or 2 by at most 1 and we do not change values at two consecutive points (other than 1, 2 and possibly 3 ) $\pi(i+1)-\pi(i)$ has the same sign as $\theta(i+1)$ $-\theta(i)$ except if $i=1$, in which case it has the opposite sign. (Note that $2 \in L_{1}$ implies that $\theta(3)-\theta(1)$ has the same sign as $\theta(3)-\theta(2)$ so the above statement holds for $i=2$.) Thus $z(\pi)=z^{-}, C_{1}(\pi)=2$, and $C_{j}(\pi)=C_{j-1}(\theta)$ for $j>1$. If $2=\theta^{a}(3), 1=\theta^{b}(3)$, then $i_{-}\left(I_{\theta}(3)\right)_{a}=$ $i_{-}\left(I_{\theta}(3)\right)_{b}=L_{2}, I_{\pi}(3)_{a}=L_{1}, I_{\pi}(3)_{b}=C_{1}$, and for $j \not \equiv a, b(\bmod n)$, $I_{\pi}(3)_{j}=i_{-}\left(I_{\theta}(3)_{j}\right)$. Let $C_{r}(\pi)$ and $C_{s}(\pi)$ be the first critical points to appear in the sequences $\pi^{-1}(2), \pi^{-2}(2), \ldots$ and $\pi^{-1}(1), \pi^{-2}(1), \ldots$, respectively. 
Assume that $L_{s-1}(\theta)$ is even, the other case being similar. Let $2=\theta^{t}\left(C_{s}(\pi)\right)$ $=\theta^{t}\left(C_{s-1}(\theta)\right)$. Then $\theta_{(s-1)[t-1]}=M_{[t-2]} L_{1}=M_{[t-1]}$, where $M$ is the maximum sequence in $I^{z}$. If $M_{[t-2]}$ were even, then $M_{[t-2]} L_{k+1}>M_{[t-2]} L_{1}$, so $M_{[t-2]}$ is odd. Hence $i_{-}\left(M_{[t-2]}\right)$ is odd in $I^{z^{-}}$, so

$$
\begin{aligned}
\left(i_{-}(F(\theta))\right)_{(s)[t-1]} & =\left(i_{-}\left(\theta_{(s-1)}\right)\right)_{[t-1]}=i_{-}\left(M_{[t-2]} L_{1}\right)=i_{-}\left(M_{[t-2]}\right) L_{2} \\
& <i_{-}\left(M_{[t-2]}\right) L_{1}=\pi_{(s)[t-1]} .
\end{aligned}
$$

A similar proof shows that $\left(i_{-}(F(\theta))\right)_{(r)}<_{r} \pi_{(r)}$.

Finally, if $l \neq r, s$ then suppose that 1 occurs before 2 in the sequence $\pi\left(C_{l}\right), \pi^{2}\left(C_{l}\right), \ldots$; the case that 2 occurs before 1 is similar. (All $C_{l}$ 's and $C_{s}$ 's are $C_{l}(\pi)$ and $C_{s}(\pi)$.) Then since this sequence differs from $\theta\left(C_{l}\right), \theta^{2}\left(C_{l}\right), \ldots$ only in that 1 and 2 are switched, we must have $I_{\pi}\left(C_{l}\right)=A I_{\pi}\left(C_{s}\right), i_{-}\left(I_{\theta}\left(C_{l}\right)\right)=$ $A i_{-}\left(I_{\theta}\left(C_{s}\right)\right)$. Then $I_{\pi}^{+}\left(C_{l}\right)=A^{\prime} I_{\pi}^{+}\left(C_{s}\right), i_{-}\left(I_{\theta}^{+}\left(C_{l}\right)\right)=A^{\prime} i_{-}\left(I_{\theta}^{+}\left(C_{s}\right)\right)$, and $A^{\prime}$ has the same parity as $l-s$. Hence $i_{-}\left(I_{\theta}^{+}\left(C_{s}\right)\right)<_{s} I_{\pi}^{+}\left(C_{s}\right)$ implies that $i_{-}\left(I_{\theta}^{+}\left(C_{l}\right)\right)<_{l} I_{\pi}^{+}\left(C_{l}\right)$.

Corollary 9.4. Suppose $\theta$ is a maximal element of $C^{n} \cap P(z), n-1 \in L_{k+1}(\theta)$, and $\left|\theta^{-1}(n-1)-\theta^{-1}(n)\right|>1$. Then there is a $\pi \in C^{n} \cap P\left(z^{+}\right)$with $\pi \rightarrow \theta$.

Proof. Apply the above lemma to $\theta^{\prime}$ defined by $\theta^{\prime}(x)=n+1-\theta(n+1-x)$.

Theorem 9.5. If $\theta \in C^{n} \cap P(z)$ and $|z|<n-2$ then there is a $\pi \in C^{n}$ with $|z(\pi)|=1+|z|$ and $\pi \rightarrow \theta$.

Proof. Since every element of $C^{n} \cap P(z)$ is dominated by a maximal one, we may assume without loss of generality that $\theta$ is maximal in $C^{n} \cap P(z)$. Then since $I_{\theta}\left(C_{1}\right)=B(\theta)$, we have $2 \in L_{1}$ or $n-1 \in L_{k+1}$. In the latter case, we can replace $\theta(x)$ with $n+1-\theta(n+1-x)$, so assume without loss of generality that $2 \in L_{1}$. If $\theta^{-1}(1)$ and $\theta^{-1}(2)$ are not consecutive, then by Lemma 9.3 we are done. So we may assume that $\theta^{-1}(1)$ and $\theta^{-1}(2)$ are consecutive. We know that $\theta^{-1}(1)=n$ or $C_{i}$ for some $i$. If $\theta^{-1}(1)=C_{i}$ and $2 \leq i \leq k-1$ then $C_{i}$ is a minimum and $\theta^{-1}(2)=C_{i-1}$ or $C_{i+1}$ which are maxima. But $\theta^{-1}(2)$ cannot be a maximum because $\theta$ takes on only one value smaller than 2. So $\theta^{-1}(1)=n, C_{1}$, or $C_{k}$.

If $\theta^{-1}(1)=C_{1}$ then $\theta^{-1}(2) \in L_{1}$ so $I_{\theta}\left(\theta^{-1}(2)\right)=L_{1} L_{1} \ldots$, so the minimum sequence in $I^{z}$ begins $L_{1} L_{1}$. Thus $L_{1}$ is even, so $C_{1}$ is a maximum. But this contradicts the fact that $\theta\left(C_{1}\right)=1$.

Similarly, if $\theta^{-1}(1)=C_{k}$, then $\theta^{-1}(2) \in L_{k+1}$, so $I_{\theta}\left(\theta^{-1}(2)\right)=L_{k+1} L_{1} \cdots$. Hence $L_{k+1}$ must be odd, so $C_{k}$ is a maximum, contradicting $\theta\left(C_{k}\right)=1$.

Finally, if $\theta^{-1}(1)=n$ then $\theta^{-1}(2)=n-1$. Since $\theta$ is one-to-one, $\theta(n-2) \geq$ 3. Thus $\theta(n-2)>2=\theta(n-1)>1=\theta(n)$, so $\theta^{-1}(2) \in L_{k+1}$. Since $n-1 \in L_{k+1}$ we can apply a similar argument to $\theta^{-1}(n)$ and $\theta^{-1}(n-1)$ to find that $\theta^{-1}(n)=1, \theta^{-1}(n-1)=2, \theta^{2}(1)=1$, a contradiction. 
Corollary 9.6. If $\theta$ is a maximal element of $C^{n}$ then $z(\theta)= \pm(n-2)$.

We will now lay the groundwork for Theorem 9.13, in which we completely describe those $\theta$ in $C^{n}$ that are maximal.

Lemma 9.7. Let $\pi, \theta \in C^{n}, z(\pi)=z(\theta)= \pm(n-2)$. Then $\pi \rightarrow \theta$ if and only if $\pi(i) \geq_{i-1} \theta(i)$ for $i=1, \ldots, n$.

Proof. Note that $C_{i}(\theta)=C_{i}(\pi)=i+1$. First suppose $\pi\left(C_{i}\right) \neq \theta\left(C_{i}\right)$. If $\pi\left(C_{i}\right)=C_{j}$ and $C_{i}$ is a minimum then points near $C_{i}$ go to points larger than $C_{j}$, so $\pi_{(i)}=L_{j+1} \cdots$. Thus if $\pi\left(C_{i}\right)=C_{j}$ and $\theta\left(C_{i}\right)=C_{l}$ then $\pi_{(i)}=L_{j+1} \cdots$ and $\theta_{(i)}=L_{l+1} \ldots$, so $\pi_{(i)}>\theta_{(i)}$ if and only if $\pi\left(C_{i}\right)>\theta\left(C_{i}\right)$. This still works if $\theta\left(C_{i}\right)$ or $\pi\left(C_{i}\right)$ is 1 since the other is at least $C_{1}$ and so the $i$ maximal shift of its fundamental sequence begins with at least $L_{2}$. Similarly, if $C_{i}$ is a maximum then $\pi_{(i)}=L_{j} \cdots$ and the same argument works. Thus if $\theta\left(C_{i}\right)>_{i} \pi\left(C_{i}\right)$ then $\theta_{(i)}>_{i} \pi_{(i)}$. Thus if $\pi \rightarrow \theta$ then $\pi(i+1) \geq_{i} \theta(i+1)$ for each $i$.

Conversely, suppose $\pi(i) \geq_{i+1} \theta(i)$ for $i=1, \ldots, n$. Suppose that for some $i, \pi_{(i)}<_{i} \theta_{(i)}$. Choose $i$ so that $\pi_{(i)}<_{i} \theta_{(i)}$ and the smallest $r$ for which $\pi_{(i) r} \neq \theta_{(i) r}$ is as small as possible. If $\pi\left(C_{i}\right)=\theta\left(C_{i}\right)=C_{j}$ then $\pi_{(j)}$ and $\theta_{(j)}$ agree to fewer terms than $\pi_{(i)}$ and $\theta_{(i)}$ so $\pi_{(j)}>_{j} \theta_{(j)}$. But then $\pi_{(i)}>_{i} \theta_{(i)}$. If $\pi\left(C_{i}\right)$ and $\theta\left(C_{i}\right)$ are different then we already know that $\pi\left(C_{i}\right)>_{i} \theta\left(C_{i}\right)$, so $\pi_{(i)}>_{i} \theta_{(i)}$. The only case remaining is $\pi\left(C_{i}\right)=\theta\left(C_{i}\right)=1$ or $n$. But then the same arguments applied to $S\left(\pi_{(i)}\right)$ and $S\left(\theta_{(i)}\right)$ show the desired inequality.

Fix $z(\theta)= \pm(n-2)$ and let $m(\theta)=m=$ the number of local minima, including endpoints that are local minima, of $\theta$, i.e.,

$$
m= \begin{cases}\lfloor(n+1) / 2\rfloor & \text { if } z(\theta)>0, \\ \lfloor n / 2\rfloor & \text { if } z(\theta)<0 .\end{cases}
$$

Let $S(\theta)=S=\{\theta(i): i$ is a local minimum, possibly including endpoints $\}$, and $T(\theta)=T=\{\theta(i): i$ is a local maximum, possibly including endpoints $\}$. Let $M(\theta)=M=$ the mixed up set of $\theta=\{x: x \in T$ and $\exists y \in S$ with $y>x$ or $x \in S$ and $\exists y \in T$ with $y<x\}$.

Lemma 9.8. If $\pi, \theta \in C^{n}, z(\pi)=z(\theta)= \pm(n-2)$, and $\pi \rightarrow \theta$, then $\pi=\phi \theta$ where $\phi$ is a permutation of $M(\theta)$. Conversely, if $\phi$ is a permutation of $M(\theta)$ such that $\phi \theta$ is cyclic, $i \in S \Rightarrow \phi(i) \leq i$ and $i \in T \Rightarrow \phi(i) \geq i$, then $\phi \theta \in C^{n}$, $z(\phi \theta)=z(\theta)$, and $\phi \theta \rightarrow \theta$.

Proof. Suppose that $\pi \rightarrow \theta$. Let $r$ be a positive integer such that $[r] \subset S$. Then $\pi\left(\theta^{-1}(1)\right) \leq 1$, so $\pi\left(\theta^{-1}(1)\right)=1, \pi\left(\theta^{-1}(2)\right) \leq 2$ so $\pi\left(\theta^{-1}(2)\right)=$ $2, \ldots, \pi\left(\theta^{-1}(r)\right)=r$. Similarly, $\pi\left(\theta^{-1}(n)\right)=n, \ldots$. So $\pi(x)=\theta(x)$ whenever $\theta(x) \subset[n] \backslash M$. Thus $\pi=\phi \theta$ for some permutation $\phi$ of $M$. Conversely, if $\phi(i) \leq i$ for $i \in S$ and $\phi(i) \geq i$ for $i \in T$ then $\phi(\theta(j)) \leq \theta(j)$ for $\theta(j) \in S$, and $\phi(\theta(j)) \geq \theta(j)$ for $\theta(j) \in T$, so $\phi \theta$ has type $z(\theta)$ and $\phi \theta \rightarrow \theta$. 
Definition. If $\theta \in S^{n}$ and $U \subset[n]$, then define $\left.\theta\right|_{U}$ to be the permutation of $U$ given by letting $\left.\theta\right|_{U}(i)=$ the element of $U$ that appears first in the sequence $\theta(i), \theta^{2}(i), \ldots$.

Lemma 9.9. If $\theta \in C^{n}$ and $\phi$ is a permutation of $U \subset[n]$ then $\phi \theta$ is cyclic if and only if $\phi\left(\left.\theta\right|_{U}\right)$ is cyclic.

Proof. Let $\left.\theta\right|_{U}=\mu, \phi \theta=\pi$. If $i \in U$, define $A_{i}$ to be the longest prefix of the sequence $i, \theta(i), \theta^{2}(i), \ldots$ that contains no element of $U$, i.e., if $r$ is the smallest positive integer with $\theta^{r}(i) \in U$ then $A_{i}=$ the sequence $i$, $\theta(i), \ldots, \theta^{r-1}(i)$. Note that $\theta^{r}(i)=\mu(i)$. Then the sequence $i, \theta(i), \ldots$, $\theta^{n-1}(i)=A_{i} A_{\mu(i)} A_{\mu^{2}(i)} \cdots A_{\mu^{|U|-1}(i)}$. Thus the sequence $i, \pi(i), \pi^{2}(i), \ldots$, $\pi^{n-1}(i)=A_{i} A_{\phi \mu(i)} A_{(\phi \mu)^{2}(i)} \cdots A_{(\phi \mu)^{|U|-1}(i)}$, so clearly $\pi$ is cyclic if and only if $\phi \mu$ is cyclic.

Lemma 9.10. If $\theta \in C^{n}, z(\theta)= \pm(n-2)$, and there are $s \in S(\theta), t, t^{\prime} \in T(\theta)$ with $t<t^{\prime}<s$, and $\left.\theta\right|_{\left\{s, t, t^{\prime}\right\}}=\left(s t t^{\prime}\right)$, then $\theta$ is not a maximal element of $C^{n}$. Proof. Let $\phi=\left(s t t^{\prime}\right)$. Then $\phi\left(\left.\theta\right|_{\left\{s, t, t^{\prime}\right\}}\right)=\left(s t^{\prime} t\right)$ is cyclic, so $\phi \theta$ is cyclic, by Lemma 9.9. Also $\phi(s)=t<s, \phi(t)=t^{\prime}>t$, and $\phi\left(t^{\prime}\right)=s>t^{\prime}$, so by Lemma $9.8 \phi \theta \rightarrow \theta$.

Lemma 9.11. If $\theta \in C^{n}, z(\theta)= \pm(n-2)$, and there are $s, s^{\prime} \in S(\theta), t \in T(\theta)$ with $t<s<s^{\prime}$, and $\left.\theta\right|_{\left\{s, s^{\prime}, t\right\}}=\left(t s^{\prime} s\right)$, then $\theta$ is not a maximal element of $C^{n}$.

Proof. The proof is essentially the same as above. Take $\phi=\left(t s^{\prime} s\right)$.

Lemma 9.12. If $\theta \in C^{n}, z(\theta)= \pm(n-2)$, and there are $s, s^{\prime} \in S(\theta), t, t^{\prime} \in$ $T(\theta)$ with $t<t^{\prime}<s<s^{\prime}$, then $\theta$ is not a maximal element of $C^{n}$.

Proof. Assume $\theta$ is maximal. By the above lemmas $\left.\theta\right|_{\left\{s, t, t^{\prime}\right\}}=\left(s t^{\prime} t\right)$, $\left.\theta\right|_{\left\{s^{\prime}, t, t^{\prime}\right\}}=\left(s^{\prime} t^{\prime} t\right),\left.\theta\right|_{\left\{s, s^{\prime}, t\right\}}=\left(s s^{\prime} t\right)$, and $\left.\theta\right|_{\left\{s, s^{\prime}, t^{\prime}\right\}}=\left(s s^{\prime} t^{\prime}\right)$. From these it is clear that $\left.\theta\right|_{\left\{s, s^{\prime}, t^{\prime}, t\right\}}=\left(s s^{\prime} t^{\prime} t\right)$. Take $\phi=\left(s t^{\prime}\right)\left(s^{\prime} t\right)$. Then $\phi(s)=$ $t^{\prime}<s, \phi\left(s^{\prime}\right)=t<s^{\prime}, \phi\left(t^{\prime}\right)=s>t^{\prime}, \phi(t)=s^{\prime}>t$, and $\phi\left(\left.\theta\right|_{\left\{s, s^{\prime}, t^{\prime}, t\right\}}\right)=$ $\left(s t^{\prime}\right)\left(s^{\prime} t\right)\left(s s^{\prime} t^{\prime} t\right)=\left(s t t^{\prime} s^{\prime}\right)$ is cyclic. Thus $\phi \theta \rightarrow \theta$ so $\theta$ is not maximal.

Theorem 9.13. Let $\theta \in C^{n}, z(\theta)= \pm(n-2)$. Let $m=m(\theta), S=S(\theta)$, $T=T(\theta)$, and $M=M(\theta)$ be defined as above. Then $\theta$ is a maximal element of $C^{n}$ if and only if

(i) $S=[m]$, or

(ii) $|S \cap[m]|=m-1$ and if $s_{1}<s_{2}<\cdots<s_{r}, t_{1}<t_{2}<\cdots<t_{s}$ are the elements of $S \cap M$ and $T \cap M$ then $\left.\theta\right|_{M}=\left(s_{1} s_{2} \cdots s_{r} t_{s} \cdots t_{2} t_{1}\right)$.

Proof. First suppose that $\theta$ is maximal. If $|S \cap[m]|=m$ then (i) holds. If $|S \cap[m]|<m-1$ then there are at least two elements of $T$ that are $\leq m$, and at least two elements of $S$ that are $>m$. By Lemma 9.12, $\theta$ is not 
maximal. Finally, if $|S \cap[m]|=m-1$, define $s_{i}$ and $t_{i}$ as in (ii). Since $t_{1}<s_{1}<s_{2}<\cdots<s_{r}$, if $\left.\theta\right|_{\left\{t_{1} s_{i} s_{i+1}\right\}}=\left(t_{1} s_{i+1} s_{i}\right)$, then by Lemma 9.11, $\theta$ is not maximal. Hence $\left.\theta\right|_{\left\{t_{1} s_{i} s_{i+1}\right\}}=\left(t_{1} s_{i} s_{i+1}\right)$. Hence $\left.\theta\right|_{\left\{t_{1}, s_{1}, s_{2}, \ldots, s_{r}\right\}}=\left(t_{1} s_{1} s_{2} \cdots s_{r}\right)$. Similarly, by Lemma 9.10, $\left.\theta\right|_{\left\{t_{1}, t_{2}, \ldots, t_{s}, s_{r}\right\}}=\left(t_{s} \cdots t_{2} t_{1} s_{r}\right)$. Combining these, we get (ii).

Conversely, suppose that $\theta$ satisfies (i) or (ii). In the former case, $\theta$ is clearly maximal. In the latter case note that $s_{1}, s_{2}, \ldots, s_{r-1} \leq m, t_{2}, t_{3}, \ldots, t_{s} \geq$ $m+1$, so $t_{1}<s_{1}<s_{2}<\cdots<s_{r-1}<t_{2}<t_{3}<\cdots<t_{s}<s_{r}$. If $\theta$ were not maximal then by Lemmas 9.7, 9.8, and 9.9, there would be a permutation of $M, \pi=\phi\left(\left.\theta\right|_{M}\right) \neq\left.\theta\right|_{M}$, that is cyclic and that satisfies $\pi\left(s_{r}\right) \geq t_{s}, \pi\left(t_{s}\right) \geq$ $t_{s-1}, \ldots, \pi\left(t_{2}\right) \geq t_{1}, \pi\left(t_{1}\right) \leq s_{1}, \pi\left(s_{1}\right) \leq s_{2}, \ldots, \pi\left(s_{r-1}\right) \leq s_{r}$. Since $\pi\left(t_{1}\right) \leq$ $s_{1}, \pi\left(t_{1}\right)=t_{1}$ or $s_{1}$. In the former case $\pi$ is not cyclic. Hence $\pi\left(t_{1}\right)=s_{1}$. If $r \geq 3$ then $\pi\left(s_{1}\right) \leq s_{2}$ implies $\pi\left(s_{1}\right)=t_{1}, s_{1}$, or $s_{2}$. Since $\pi\left(t_{1}\right)=s_{1}$, $\pi\left(s_{1}\right) \neq\left(s_{1}\right)$. If $\pi\left(s_{1}\right)=t_{1}$ then $\pi^{2}\left(s_{1}\right)=s_{1}$, so $\pi$ is not cyclic. Hence $\pi\left(s_{1}\right)=s_{2}$. Continuing in this way we see that $\pi\left(s_{i}\right)=s_{i+1}$ for $i \leq r-2$. Likewise $\pi\left(s_{r}\right)=t_{s}$ and $\pi\left(t_{i}\right)=t_{i-1}$ for $i \geq 3$. This determines $\pi$ except for $\pi\left(s_{r-1}\right)$ and $\pi\left(t_{2}\right)$, which must be $t_{1}$ and $s_{r}$ in some order. If $\pi\left(s_{r-1}\right)=s_{r}$ then $\pi=\left.\theta\right|_{M}$. Otherwise, $\pi\left(s_{r-1}\right)=t_{1}$ which implies $\pi^{r}\left(t_{1}\right)=t_{1}$, contradicting the fact that $\pi$ is cyclic.

Remark. We can use the ideas from the proof of Theorem 9.13 to obtain necessary and sufficient conditions for a permutation $\theta$ to be a maximal element of $C^{n} \cap P(z)$.

We already know from Corollary 9.2 that $I_{\theta}\left(C_{1}\right)=B(\theta)$ for any such $\theta$. As in the definition of $B(\theta)$ we set $I_{\theta}\left(C_{1}\right)=\left(C_{i_{1}} B_{1} C_{i_{2}} B_{2} \cdots C_{i_{k}} B_{k}\right)^{\infty}$. Clearly, if $\pi \in P(z)$ and $\pi \rightarrow \theta$ then for each $i$, the string of $L$ 's following $C_{i}$ in $I_{\pi}\left(C_{1}\right)$ must be at least as long as the corresponding string in $I_{\theta}\left(C_{1}\right)$. If $\pi \in C^{n}$, then these two strings must have the same length. Thus $I_{\pi}\left(C_{1}\right)$ is formed by permuting the "blocks" $C_{i_{j}} B_{j}$.

Let $\phi \in C^{k}$ be defined by $\phi\left(i_{j}\right)=i_{j+1}$ for $1 \leq j \leq k-1, \phi\left(i_{k}\right)=i_{1}$. Define $i_{j}$ to be a pseudomaximum if $C_{i_{j}}$ is a maximum and $B_{j}$ is even, or $C_{i_{j}}$ is a minimum and $B_{j}$ is odd. Define $i_{j}$ to be a pseudominimum otherwise. Let $m=$ the number of pseudominima, $S=\{\phi(i): i$ is a pseudominimum $\}$, and define $T$ and $M$ likewise.

Then a permutation $\theta$ is maximal in $C^{n} \cap P(z)$ if and only if $\phi, m, S, T$, and $M$ satisfy condition (i) or (ii) of Theorem 9.13.

Acknowledgment. The author would like to thank Persi Diaconis for suggesting this problem.

\section{REFERENCES}

1. S. Baldwin, Generalizations of a theorem of Sarkovskii on orbits of continuous real-valued functions, preprint.

2. Chris Bernhardt, The ordering on permutations induced by continuous maps of the real line, preprint. 
3. L. Block, Simple periodic orbits of mappings of the interval, Trans. Amer. Math. Soc. 254 (1979), 391-398.

4. L. Block and W. Coppel, Stratification of continuous maps of an interval, preprint.

5. L. Block and D. Hart, Stratification of the space of unimodal interval maps, preprint.

6. P. Collet and J. Eckmann, Iterated maps on the interval as dynamical systerrus, Progress in Physics, vol. 1, Birkhäuser, Boston, Mass., 1980.

7. J. Guckenheimer, Bifurcations of dynamical systems, in Dynamical Systems (C.I.M.E. Lectures, Bressanone, Italy, June 1978), Progress in Math., vol. 8., Birkhäuser, Boston, Mass., 1980.

8. C. W. Ho, On the structure of minimum orbits of periodic points for maps of a real line, preprint.

Department of Mathematics, Harvard University, Cambridge, Massachusetts 02138 